\title{
Development of a cost-effective metabarcoding strategy for analysis of the marine phytoplankton community
}

Tae-Ho Yoon, Hye-Eun Kang, Chang-Keun Kang, Sang Heon Lee, Do-Hwan Ahn, Hyun Park, Hyun-Woo Kim

We developed a cost-effective metabarcoding strategy to analyze phytoplankton community structure using the Illumina MiSeq system. The amplicons (404 - 411 bp) obtained by end-pairing of two reads were sufficiently long to distinguish algal species and provided barcode data equivalent to those generated with the Roche 454 system, but at less than 1/20th of the cost. The original universal primer sequences targeting the $23 \mathrm{~S}$ rDNA region and the PCR strategy were both modified, and this resulted in higher numbers of eukaryotic algal sequences by excluding non-photosynthetic proteobacterial sequences supporting effectiveness of this strategy. The novel strategy was used to analyze the phytoplankton community structure of six water samples from the East/Japan Sea: surface and $50 \mathrm{~m}$ depths at coastal and open-sea sites, with collections in May and July 2014. In total, 345 operational taxonomic units (OTUs) were identified, which covered most of the prokaryotic and eukaryotic algal phyla, including Dinophyta, Rhodophyta, Ochrophyta, Chlorophyta, Streptophyta, Cryptophyta, Haptophyta, and Cyanophyta. This highlights the importance of plastid $23 \mathrm{~S}$ primers, which perform better than the currently used $16 \mathrm{~S}$ primers for phytoplankton community surveys. The findings also revealed that more efforts should be made to update $23 \mathrm{~S}$ rDNA sequences as well as those of $16 \mathrm{~S}$ in the databases. Analysis of algal proportions in the six samples showed that community structure differed depending on location, depth and season. Across the six samples evaluated, the numbers of OTUs in each phylum were similar but their relative proportions varied. This novel strategy would allow laboratories to analyze large numbers of samples at reasonable expense, whereas this has not been possible to date due to cost and time. In addition, we expect that this strategy will generate a large amount of novel data that could potentially change established methods and tools that are currently used in the realms of oceanography and marine ecology. 
2 Development of a cost-effective metabarcoding strategy for analysis of the marine

\section{3 phytoplankton community}

4

5 Tae-Ho Yoon ${ }^{\mathrm{a}}$, Hye-Eun Kang ${ }^{\mathrm{b}}$, Chang-Keun Kang ${ }^{\mathrm{c}}$, Sang Heon Lee ${ }^{\mathrm{e}}$, Do Hwan Ahn ${ }^{\mathrm{d}}$, Hyun Park

6 and Hyun-Woo Kim ${ }^{\text {ab* }}$

7

8 a Interdisciplinary Program of Biomedical Engineering, Pukyong National University, Busan,

9 608-737, South Korea

10 b Department of Marine Biology, Pukyong National University, Busan 608-737, South Korea

$11{ }^{\mathrm{c}}$ School of Environmental Science \& Engineering, Gwangju Institute of Science and

12 Technology, Gwangju 500-712, South Korea

13 ' Korea Polar Research Institute, Korea Ocean Research and Development Institute, Incheon 406-

14840 , South Korea

15 eDepartment of Oceanography, Pusan National University, Busan 609-735, South Korea

16

17 Keywords: next-generation sequencing, barcode, phytoplankton, genomics, oceanography

Dr. Hyun Park

* Corresponding authors:

Dr. Hyun-Woo Kim

Department of Marine Biology

Pukyong National University

Busan 608-737, South Korea

Tel: 82-51-629-5926

Fax: 82-51-629-5930

e-mail: kimhw@pknu.ac.kr

Korea Polar Research Institute

213-3, Songdo-dong, Yeonsu-gu,

Incheon 406-840, South Korea

34 Tel: $+82-32-760-5570$

35 Fax: +82-32-760-5575

36 e-mail: hpark@kopri.re.kr 
38

39

40

41

42

43

44

45

\section{Abstract}

We developed a cost-effective metabarcoding strategy to analyze phytoplankton community structure using the Illumina MiSeq system. The amplicons (404 - $411 \mathrm{bp}$ ) obtained by end-pairing of two reads were sufficiently long to distinguish algal species and provided barcode data equivalent to those generated with the Roche 454 system, but at less than $1 / 20$ th of the cost. The original universal primer sequences targeting the $23 \mathrm{~S}$ rDNA region and the PCR strategy were both modified, and this resulted in higher numbers of eukaryotic algal sequences by excluding non-photosynthetic proteobacterial sequences supporting effectiveness of this strategy. The novel strategy was used to analyze the phytoplankton community structure of six water samples from the East/Japan Sea: surface and $50 \mathrm{~m}$ depths at coastal and open-sea sites, with collections in May and July 2014. In total, 345 operational taxonomic units (OTUs) were identified, which covered most of the prokaryotic and eukaryotic algal phyla, including Dinophyta, Rhodophyta, Ochrophyta, Chlorophyta, Streptophyta, Cryptophyta, Haptophyta, and Cyanophyta. This highlights the importance of plastid 23S primers, which perform better than the currently used $16 \mathrm{~S}$ primers for phytoplankton community surveys. The findings also revealed that more efforts should be made to update $23 \mathrm{~S}$ rDNA sequences as well as those of $16 \mathrm{~S}$ in the databases. Analysis of algal proportions in the six samples showed that community structure differed depending on location, depth and season. Across the six samples evaluated, the numbers of OTUs in each phylum were similar but their relative proportions varied. This novel strategy would allow laboratories to analyze large numbers of samples at reasonable expense, whereas this has not been possible to date due to cost and time. In addition, we expect that this strategy will generate a large amount of novel data that could potentially change established methods and tools that are currently used in the realms of oceanography and marine ecology. 

require significant time and trained experts to obtain accurate data. This has created a bottleneck and is a major consideration in research planning. For these reasons, there is a need for a faster and more reliable standard method of performing detailed analysis of phytoplankton community structures. Alternatively, phytoplankton communities can be roughly analyzed based on different pigments using flow-cytometry (Dubelaar et al., 2007), which can sometimes be integrated with digital imaging systems (Campbell et al., 2010; Kachel and Wietzorrek, 2000; Olson et al., 2003). However, such strategies aimed at replacing microscopic observations are still far from reliable for discriminating the numerous phytoplankton taxa. are now being applied. The most widely used DNA markers include two protein-coding genes, protein D1 of photosystem-II reaction center (psbA) and a large subunit of the ribulose-1,5diphophate carxoylase/oxygenase (rbcL) or plastid 16S rDNA region (Kirkham et al., 2013; Man-

77 Aharonovich et al., 2010; McDonald et al., 2007; Paul et al., 2000; Zeidner et al., 2003). In 78 particular, the $16 \mathrm{~S}$ rDNA sequence has been well documented in the GenBank database 79 (http://www.m.nih.gov/genbank/) and more than 6,490 phytoplanktonic 16S sequences are currently deposited in the PhytoREF database (http://phytoref.org). Although there is still debate, universal primers targeting 16S rDNA are now most widely used (Decelle et al., 2015; Herlemann et al., 2011). However, it has been difficult to design a universal primer set that will amplify all 83 phytoplankton taxa from cyanobacteria to eukaryotic algae in the 16S rDNA region, and most 84 studies have analyzed specific taxonomic groups, especially for the bacterial communities (Asudi 85 et al., 2016; Cruaud et al., 2014; Kitamura et al., 2016; Le Bescot et al., 2015; Logares et al., 2014; 
86 Massana et al., 2015; Valenzuela-González et al., 2016; Vierheilig et al., 2015). Although the

87 sequence information from the $23 \mathrm{~S}$ rDNA region is only a subset of that for $16 \mathrm{~S}$ rDNA in the 88 database, this region is considered an important marker for phytoplankton community structure 89 when designing better universal primer sets that will cover most phytoplankton taxa (Folmer et al.,

90

91

92

93

94

95

96

97

98

99

100

101

102

103

104

105

106

107

108

1994; Sherwood and Presting, 2007).

The rapid pace of technological advancement in DNA sequencing and bioinformatics analysis has meant that researchers can now analyze phytoplankton community structure simply by massive sequencing of DNA barcodes that are amplified from environmental samples, a process known as "metabarcoding." In fact, several trials have already been conducted to analyze algal community structure using next generation sequencing (NGS) technology and generating large amounts of data for large survey areas at low cost, as compared with the Sanger method which uses dideoxynucleotide analogs (Eiler et al., 2013; Steven et al., 2012). These studies were dependent on the Roche 454 platform because its maximum read length (approximately $700 \mathrm{bp}$ ) is sufficient to identify to the species level. However, the cost for sequencing with the Roche 454 system remains high, and the Illumina platform has been introduced to replace this system and produce the same amount of sequence information at less than $1 / 100^{\text {th }}$ of the cost (Luo et al., 2012). In fact, virtually all studies of microbial community structure are now being conducted using the Illumina sequencer (Caporaso et al., 2012; Degnan and Ochman, 2012; Jiang et al., 2013; Logares et al., 2014; Schmidt et al., 2013; Shakya et al., 2013; Staley et al., 2013) Still, phytoplankton community structure analysis using the Illumina system has yet to be widely applied, mainly due to the lack of universal primers that are suitable for the Illumina sequencer.

In this study, we developed a novel strategy of targeting the $23 \mathrm{~S}$ rDNA region for phytoplankton community analysis using the MiSeq system (Illumina, USA). Performing large 
109 reads (404 to $411 \mathrm{bp}$ ) by paired-end sequencing (2X $300 \mathrm{bp)} \mathrm{enabled} \mathrm{us} \mathrm{to} \mathrm{analyze} \mathrm{phytoplankton}$

110 community structure at reduced cost (less than $1 / 20^{\text {th }}$ of the cost with the Roche 454 system) and

111 with high resolution. Our modified "universal primers" targeting the 23S rDNA region achieved a

112 high level of coverage, amplifying most taxa of prokaryotic and eukaryotic phytoplankton while

113 excluding the non-photosynthetic proteobacterial sequences. The reliability of this NGS strategy

114 was confirmed by analyzing phytoplankton samples from different locations, depths, and seasons

115 in the East/Japan Sea.

116

117

Materials and Methods

118

119

Sample collection and DNA extraction

120

Sampling was carried out in May and July 2014 under permitting (Department of Fisheries

121 Resource Management-1728 \& 2506) for the project "Long-term change of structure and function

122 in marine ecosystems of Korea," funded by the Ministry of Oceans and Fisheries, Korea. Field

123 water samples from the surface $(0 \mathrm{~m})$ and at $50 \mathrm{~m}$ depth were collected using $10 \mathrm{~L}$ Niskin bottles

124 at two sampling stations in the southwestern East/Japan Sea, Korea. Among various sample site

125 options, station I (StI) was chosen as representative coastal water, whereas station II (StII) was in

126 open sea. Samples were labeled with collection date, location, and depth, as follows: MIS: surface

127 water from station I in May 2014 Station I surface water; MIIS: surface water from station II in

128 May 2014; MIID: $50 \mathrm{~m}$ water from station 2 in May 2014; JIS: surface water from station I in July

129 2014; JIIS: surface water from station II in July 2014.

130 Water temperature and salinity were measured using an SBE-43 unit (Sea-Bird Electronics,

131 USA). One liter of water was collected from each sampling site and filtered using a $0.45-\mu \mathrm{m} \mathrm{GH}$ 
132 Polypro membrane filter (Pall Corporation, USA). Chlorophyll- $a$ concentration was measured

133 using a Turner 10AU fluorometer (Turner Design Co., USA) as described by Parson et al. (1984).

134 The filtered sample was ground into a fine powder using a mortar and pestle with liquid nitrogen.

135 The total DNA from the homogenized phytoplankton was extracted using a DNeasy ${ }^{\circledR}$ plant mini

136 kit (Qiagen, Germany) following the manufacturer's instructions. Extracted DNA was quantified

137 and qualified using a NanoDrop spectrophotometer ND-1000 (Thermo Scientific, USA) and was

138 stored at $-70^{\circ} \mathrm{C}$ until it was used.

139

140 Library preparation and sequencing

141 The "universal" primers for the mini-barcode were redesigned to target the plastid 23S rDNA

142 region (Sherwood and Presting, 2007). According to multiple sequence alignments of 1,683 23S

143 rDNA sequences (545 from eukaryotes and 1,138 from prokaryotes), several variations were

144 detected in the forward primer. Based on these findings, the forward primer to be used were

145 modified to increase specificity for phytoplankton taxa (Table 1). To lower the PCR-based bias, a

146 nested PCR strategy with low cycle numbers was adopted. Two forward primers

147 (A23SrVF1:GGACARAAAGACCCTATG and A23SrVF2: CARAAAGACCCTATGMAGCT)

148 and two reverse primers (A23SrVR1:AGATCAGCCTGTTATCC and A23SrVR2:

149 TCAGCCTGTTATCCCTAG) were designed and synthesized (Macrogen, Republic of Korea).

150 The first and second PCR amplifications were performed under the following cycling conditions:

151 initial denaturation at $94^{\circ} \mathrm{C}$ for $3 \mathrm{~min}$, followed by 15 cycles at $94^{\circ} \mathrm{C}$ for $30 \mathrm{~s}, 55^{\circ} \mathrm{C}$ for $30 \mathrm{~s}$, and

$15272^{\circ} \mathrm{C}$ for $30 \mathrm{~s}$, with a final extension at $72^{\circ} \mathrm{C}$ for $3 \mathrm{~min}$. The first PCR reaction mixture $(40 \mu \mathrm{L})$

153 contained $10 \mathrm{ng}$ of template, $2 \mu \mathrm{L}$ of each primer $(20 \mathrm{pmol}), 4 \mu \mathrm{L}$ of dNTPs $(10 \mathrm{mM}), 0.4 \mu \mathrm{L}$ Ex

154 Taq Hot Start Version (2 U) (Takara Bio Inc., Japan), and $4 \mu \mathrm{L} 10 \mathrm{X}$ buffer. The first amplicon 
155 was purified using the AccuPrep ${ }^{\circledR}$ Gel Purification Kit (Bioneer, Republic of Korea) and eluted

156 with $20 \mu \mathrm{L}$ elution buffer. The second PCR reaction mixture was the same as the first except that

$1572 \mu \mathrm{L}$ of purified PCR product from the first PCR amplification was used. As with the first round,

$1582 \mu \mathrm{L}$ of each primer was used for the second PCR ( $20 \mathrm{pmol})$. The products were separated by $1.5 \%$ 159 agarose gel electrophoresis and stained with loading star (Dynebio, Republic of Korea). Amplicons 160 of the expected sizes (approximately $410 \mathrm{bp}$ ) were purified using the AccuPrep ${ }^{\circledR}$ Gel Purification

161 Kit (Bioneer, Republic of Korea). A library was constructed from the NGS data using the TruSeq ${ }^{\circledR}$

162 Sample Preparation kit V2 (Illumina, USA). The quality and quantity of the library were measured 163 using a 2100 Bioanalyzer (Agilent Technologies, USA) and sequencing was performed using 164 Illumina MiSeq (2 X 300 bp pair-ends) (Illumina, USA).

Data processing of phytoplankton sequence data

167 Adapter/index and QV $<20$ sequences from the raw data obtained were trimmed, and read 168 length of $<120$ nucleotides were filtered using CLC Genomics Workbench v. 8.0 (CLC Bio, USA).

169 End-paired amplicons were constructed using Mothur software v. 1.35.0 (Schloss et al., 2009) with

170 the above 6 bp overlapping sequences and omitting any mismatches. The primer sequences were

171 trimmed using the pdiffs $=0$ option and Mothur software v 1.35.0. The PHYTOGEN database was

172 constructed and updated as shown in Figure 1. First, 3,581 sequences from the GenBank database

173 (http://www.ncbi.nlm.nih.gov/) showing high similarity (>90\%) to the amplicons obtained were

174 included in the database. "Uncultured" sequences that had been deposited in GenBank by

175 barcoding or metabarcoding projects were excluded. If the end-paired amplicons matched $100 \%$

176 with sequences in the PHYTOGEN database after alignment using BLASTn, the amplicons were

177 named. If they did not match, the amplicons were named as Class name + PKNUE + numbers and 
178 entered into the PHYTOGEN database. To avoid errors, operational taxonomic units (OTUs) were

179 not assigned for amplicons that were present in $<0.1 \%$ of the community. Once the PHYTOGEN

180 database was constructed, the total amplicons were analyzed by a BLASTn search and those with

$181<99 \%$ identity in the PHYTOGEN database were described as "others."

182

183

\section{Results}

184

185

186

\section{Evaluation of the modified algal plastid $23 S$ rDNA universal primers}

As noted, the first step of the study was to modify the original "universal" primers targeting

187 the plastid 23S rDNA region such that they excluded the non-photosynthetic proteobacteria (Table

188 1). Amplicons obtained from between the original universal primers and between the modified 189 primers were compared (Table 2). In total, 185,773 (63 OTUs) and 625,917 (106 OTUs) amplicons 190 were obtained using the original and modified primers, respectively (Table 2). Thirty-three OTUs

191 were commonly identified by both primers. Thirty OTUs were exclusively identified from the 192 sequence data generated with original universal primers, and all were proteobacteria. Seventy193 three OTUs were exclusively identified from the sequence data generated with the modified 194 primers, and all were eukaryotic algae. The difference in OTU numbers indicated that the 195 taxonomic proportions of amplicons differed between the two sets of sequence data. Regarding 196 the amplicons generated using the original universal primers, more than $84 \%$ were either unknown $197(43.9 \%)$ or proteobacteria $(41.06 \%)$ according to the sequence data (Table 2). In contrast, 198 regarding the amplicons generated using the modified primers, less than $24 \%$ were unknown $199(19.52 \%)$ or proteobacteria (4.55\%), which reflected the presence of more algal amplicons and the 200 elimination of non-photosynthetic proteobacteria (Table 2). The proportions of eukaryotic algal 
201 sequences belonging to the Haptophyta and Dinophyta were also greater when the modified

202 primers were used. Collectively, the original universal primers were improved by the 203 modifications that were made to the sequences.

204

\section{Construction of the algal ribosomal large subunit metabarcode database}

206 To determine whether the modified 23S primers and PCR conditions could be directly applied 207 to the phytoplankton survey, the described six samples were chosen and analyzed according to 208 the different locations, seasons, and depths of the East/Japan Sea. After processing the raw reads, 209 the number of end-paired amplicons obtained from each sample ranged from 290,082 to 639,836, and averaged 438,896 (Table 2). After several data processing steps, 345 OTUs were obtained

211 from six samples combined (Table 2). Despite relatively small numbers of OTUs, only 95 (27.5\%) matched the sequences previously listed in the GenBank database, indicating much lower numbers of the plastid 23S rDNA sequences compared with those of $16 \mathrm{~S}$ rDNA in the database. In addition to the 95 OTUs identified, the remaining 250 OTUs were added to the PHYTOGEN database after assigning taxonomic ranks (phyla and classes) based on sequence similarity.

216 To determine the cut-off value for phylum similarity, the OTUs obtained were screened from $21790 \%$ to $99 \%$ (Fig. 2). The data revealed minimal differences in phyla between $90 \%$ and $97 \%$, 218 whereas the presence of significant unclassified OTUs increased as the cut-off was shifted $97 \%$ to $99 \%$ (Fig. 2). Considering this, the optimal cut-off for similarity was set at $97 \%$ for assigning 220 the phylum of the OTUs. This indicated that the modified primers successfully amplified most 221 phyla of the eurkayotic and prokaryotic algae in the samples, including the Dinophyta, 222 Rhodophyta, Ochrophyta, Chlorophyta, Streptophyta, Cryptophyta, Haptophyta, and Cyanophyta 223 (Fig. 3). Phyla not found in this study, such as Bigyra, Ciliophora, Katablepharidophyta, and 
224 Telonemia, either had ambiguous taxonomies or were rare at our sample sites. Sequences that had

$225<97 \%$ identity with the PYTOGEN database were classified as "unknown," and the proportions

226 in this category ranged from $2.34 \%$ to $17.03 \%$ depending on the sample (Table 3 ). To ascertain

227 whether further analysis of the OTUs at class level was possible, similarity from $97 \%$ to $100 \%$

228 was tested (Fig. 2B). While there was considerable difference from $98 \%$ to $100 \%$, no significant

229 difference was identified below $98 \%$, indicating that $98 \%$ was the optimal similarity cut-off point

230 for assigning class. We were unable to assign family level or below mainly due to the relatively

231 small numbers of plastid $23 \mathrm{~S}$ sequences in the database.

232

233 Community structure analysis for phytoplankton of the East/Japan Sea

234 More OTUs were present in the samples collected from the open sea than in those collected 235 from the coastal seas, and this was mainly to the prokaryotic population, including cyanobacteria 236 and proteobacteria (Table 3). The costal surface water sample in July (JIS) contained the lowest 237 number of OTUs (266), whereas the open surface water sample in July (JIIS) contained the highest 238 number of OTUs (325) (Table 3). The numbers of exclusively identified OTUs at each sample site 239 were very low, ranging from 10 to 24 (approximately 5\% of the total OTUs [Fig. 4B]). The 240 numbers of OTUs in each phylum were similar in each sample, and only their relative proportions 241 varied (Table 3). For example, the numbers of OTUs belonging to the Ochrophyta were similar 242 (109 to 123) among the six samples, but the corresponding abundance proportion varied from $2437.32 \%$ to $86.40 \%$. Species belonging to the Ochrophyta comprised $>80 \%$ of those identified in the 244 coastal water samples collected in May and July (Table 3), and most belonged to class 245 Coscinodiscophyceae (Table 4). The surface and deep water samples from the open sea exhibited 246 very different populations of cyanobacteria and proteobacteria (Table. 3). Cyanobacteria occupied 
247 approximately $20 \%$ of the phytoplankton community in the surface water of the open sea samples

248 from May and July, whereas the corresponding proportions in the coastal water samples were both

$2491 \%$. Although the proportions of algal communities in the coastal surface waters and those in the

250 deep waters of the open sea were similar at the different sampling times, a considerable difference

251 was detected in the algal communities of the surface water of the open sea between May and July

252 (Table 3). The proportion of species belonging to phylum Chlorophyta increased from $2.3 \%$ in the

253 May open-sea surface water sample to $23.91 \%$ in July, whereas those in phylum Ochrophyta

254 decreased from $36.66 \%$ in May to $7.32 \%$ in July (Table 3). The major differences between the

255 May and July open-sea deep water samples were increases in proportions of species belonging to 256 the Dinophyta (from $2.33 \%$ to $13.01 \%$ ) and Ochrophyta (from $17.74 \%$ to $28.48 \%$ ) (Fig. 2).

257

258

259

260

261

262

263

264

265

266

267

268

269

\section{Discussion}

\section{Reliability of the modified universal primers for algal community structure analysis}

The modified universal primers were more effective at identifying prokaryotic and eukaryotic phytoplankton taxa, and effectively excluded proteobacterial sequences. Compared with $16 \mathrm{~S}$ primers, the modified primers will be more useful for analyzing phytoplankton community structure, as they allow for identification of more photosynthetic phytoplankton. When data produced by both $16 \mathrm{~S}$ and $23 \mathrm{~S}$ primers are compared, it will be possible to assess for correlations between autotrophic phytoplankton species and heterotrophic bacteria in the ocean. In the present study, we also found that all the sequences belonging to the Chlorophyta contained a GGACAA sequence rather than GGACAG at the 5' end of the original universal forward primer region (Table 1). This finding enabled us to modify the primers such that we were able to detect 
270 a greater proportion of Chlorophyta in the surface water of the open sea, and this supports the

271 value of the modified universal primers (Fig. 4). In addition, the nested PCR using a low cycle

272 number and modified universal primers yielded more eukaryotic algal sequences than the original

273 primers did. Use of a single primer set often results in exaggerated stochastic effects due to the

274 exponential nature of PCR amplification and variable primer efficiency (Caragine et al., 2009;

275 Pinto and Raskin, 2012). The original 23S primers, which cannot discriminate heterotrophic

276 proteobacteria from other photosynthetic phytoplankton species (whereas 16S primers can do so),

277 yielded fewer eukaryotic algal sequences when the protobacterial population was large (Table 2).

278 To overcome those drawbacks and to present better quantitative phytoplankton community

279 structure, it is necessary to use reduced PCR cycles and different primer sets. In the present study,

280 a nested PCR strategy with low cycle number reduced the stochastic effects of PCR and yielded

281 more photosynthetic algal species sequences presenting the better phytoplankton community

282 structure for each sample examined. In conclusion, the performance of the modified 23S universal

283 primers was superior to that of the original universal primers designed for marine phytoplankton

284 community structure analysis.

285 Of the 345 OTUs identified in this study, only 95 (27.5\%) matched the sequences previously listed

286 in the GenBank database, and this is an important limitation of the $23 \mathrm{~S}$ primers. This is mainly

287 because most phytoplankton researchers have paid more attention to the molecular taxonomy of

288 the $16 \mathrm{~S}$ rDNA region than the $23 \mathrm{~S}$ rDNA region. Although many more $16 \mathrm{~S}$ rDNA sequences have

289 been deposited in the database, it is difficult to design the "universal primers" that will amplify all

290 the prokaryotic and eukaryotic algal taxa that correspond to the $16 \mathrm{~S}$ rDNA region. In fact, most

291 studies that have used the universal primers have been conducted to determine the biodiversity of

292 a specific taxon or group (Asudi et al. 2016; Cruaud et al. 2014; Kitamura et al. 2016; Logares et 
293 al. 2014; Valenzuela-González et al. 2016; Vierheilig et al. 2015). In contrast, our current results

294 with the modified primers that target plastid 23S rDNA indicate that these can be used as "universal

295 primers" for analyzing algal community structure because of their large coverage and the fact that

296 they amplify both prokaryotic and eukaryotic algal species. For this reason, if PHYTOGEN is

297 operated properly documenting the plastid 23S rDNA sequences this would be a useful database

298 for understanding the marine phytoplankton community. Without doubt, the first step to improving

299 the PHYTOGEN database would be to increase the sequence data with species description. Since 300 a great deal of $16 \mathrm{~S}$ rDNA data have already exist, one of the fastest ways to increase the sequence 301 information is to perform massive sequencing the long DNA fragments that contain both $16 \mathrm{~S}$ 302 rDNA and 23S rDNA regions within the same sample. Given that the cost for sequencing is 303 steadily decreasing, it is expected that the availability of plastid 23S rDNA sequences will increase 304 dramatically and this will enable researchers to ultimately obtain extensive, useful 23S rDNA data 305 without any further morphological analysis. In addition, the PHYTOGEN database will need to be 306 updated periodically to add new findings for $23 \mathrm{~S}$ rDNA to the GenBank database. Once 307 PHYTOGEN has been updated and higher resolution data become available, we will produce data 308 with better resolution and our dependence on molecular surveys for marine phytoplankton 309 evaluations will increase.

310

311 Effects of economic metabarcording strategy on marine phytoplankton studies

312 After processing the raw reads, the numbers of end-paired amplicons obtained from each 313 respective sample ranged from 290,082 to 639,836, and averaged 438,896 (Table 3). Compared 314 with the previously reported average amplicon numbers produced by the Roche 454 315 pyrosequencing system (Eiler et al., 2013), equivalent data have been obtained at less than $1 / 100^{\text {th }}$ 
316 of the cost using the Illumina platform (Mardis, 2008). Based on this, several studies have since

317 used the MiSeq platform to analyze bacterial community structure using 16S primers (Caporaso

318 et al., 2012; Schmidt et al., 2013); however, "universal" primers that are suitable for the Illumina

319 platform (400 bp - $500 \mathrm{bp}$ ) and cover prokaryotic and eukaryotic phytoplankton have not been

320 reported. The modified primers we designed for this study are not only optimized for the Illumina

321 platform with high resolving length (404 to $411 \mathrm{bp}$ ), but also exhibit large taxonomic coverage,

322 amplifying prokaryotic as well as eukaryotic phytoplankton. In our present study, compared to

323 the Roche 454 system, we obtained similar data at 1/20th the cost using the Illumina MiSeq

324 system after trimming the raw data with high quality (6 bp overlapping sequences and omitting

325 any mismatches). The cost would be further reduced to reach a similar conclusion by the increased

326 indices or the less strict trimming algorithm. Considering the low cost of the metabarcoding

327 strategy, it is possible for a single laboratory to run a year-round phytoplankton community survey

328 handling the several thousand samples, which have never imagined. This means that researchers

329 should spend more time in the laboratory analyzing the huge data using various bioinformatics

330 tools instead of collecting samples in the field.

331 This economic metabarcoding strategy enables large amounts of data to be analyzed at one

332 time and will allow researchers to obtain novel knowledge. We recognize that certain features of

333 the sampling methods should be changed, specifically, the filtered water volume should be $10 \mathrm{~L}$

334 per sample, as opposed to the $0.5 \mathrm{~L}$ volume used in our phytoplankton survey. A previous study

335 showed that OTU richness was greater with increased filtered water volume (Padilla et al., 2015);

336 therefore, increasing the water volume per sample would generate more accurate results, less

337 variability and higher OTU numbers. If the proportion of each OTU is linked to the total carbon

338 values, we would also be able to estimate the carbon biomass of each OTU. We could construct a 
339 spatio-temporal database of phytoplankton community changes on a daily or even hourly basis.

340 Most of all, this new strategy would be especially useful for analyzing phytoplankton community

341 structure and its contribution to primary production in ocean. One long-term question is whether

342 picoplankton are expected to gradually increase as the oceans become warmer (Morán et al., 2010).

343 These data would provide novel information about the paradoxical nature of phytoplankton

344 (Hutchinson, 1961) and its ecosystem functions in marine food webs, which have not been

345 investigated to date.

346 
Asudi, G.O., Van den Berg, J., Midega, C.A., Schneider, B., Seemüller, E., Pickett, J.A., Khan, Z.R., 2016. Detection, Identification, and Significance of Phytoplasmas in Wild Grasses in East Africa. Plant Disease 100, 108-115.

Campbell, L., Olson, R.J., Sosik, H.M., Abraham, A., Henrichs, D.W., Hyatt, C.J., Buskey, E.J., 2010. FIRST HARMFUL DINOPHYSIS (DINOPHYCEAE, DINOPHYSIALES) BLOOM IN THE US IS REVEALED BY AUTOMATED IMAGING FLOW CYTOMETRY1. Journal of Phycology 46, 66-75.

Caporaso, J.G., Lauber, C.L., Walters, W.A., Berg-Lyons, D., Huntley, J., Fierer, N., Owens, S.M., Betley, J., Fraser, L., Bauer, M., 2012. Ultra-high-throughput microbial community analysis on the Illumina HiSeq and MiSeq platforms. The ISME journal 6, 1621-1624. Validation of testing and interpretation protocols for low template DNA samples using AmpFISTR Identifiler. Croatian medical journal 50, 250-267.

Cruaud, P., Vigneron, A., Lucchetti-Miganeh, C., Ciron, P.E., Godfroy, A., Cambon-Bonavita, M.-A., 2014. Influence of DNA extraction method, 16S rRNA targeted hypervariable regions, and sample origin on microbial diversity detected by 454 pyrosequencing in marine chemosynthetic ecosystems. Applied and environmental microbiology 80, 4626-4639.

366

367

368

369

370

371

372

373

374

375

376

377

378

379

380

381

382

383

Decelle, J., Romac, S., Stern, R.F., Bendif el, M., Zingone, A., Audic, S., Guiry, M.D., Guillou, L., Tessier, D., Le Gall, F., Gourvil, P., Dos Santos, A.L., Probert, I., Vaulot, D., de Vargas, C., Christen, R., 2015. PhytoREF: a reference database of the plastidial 16S rRNA gene of photosynthetic eukaryotes with curated taxonomy. Molecular ecology resources 15, 1435-1445.

Degnan, P.H., Ochman, H., 2012. Illumina-based analysis of microbial community diversity. The ISME journal 6, 183-194.

Dubelaar, G.B.J., Casotti, R., Tarran, G.A., Biegala, I.C., 2007. Phytoplankton and their Analysis by Flow Cytometry, Flow Cytometry with Plant Cells. Wiley-VCH Verlag GmbH \& Co. KGaA, 287-322.

Eiler, A., Drakare, S., Bertilsson, S., Pernthaler, J., Peura, S., Rofner, C., Simek, K., Yang, Y., Znachor, P., Lindström, E.S., 2013. Unveiling Distribution Patterns of Freshwater Phytoplankton by a Next Generation Sequencing Based Approach. PLoS ONE 8, e53516.

Folmer, O., Black, M., Hoeh, W., Lutz, R., Vrijenhoek, R., 1994. DNA primers for amplification of mitochondrial cytochrome $\mathrm{c}$ oxidase subunit I from diverse metazoan invertebrates. Molecular marine biology and biotechnology 3, 294-299.

Herlemann, D.P., Labrenz, M., Jurgens, K., Bertilsson, S., Waniek, J.J., Andersson, A.F., 2011. Transitions in bacterial communities along the $2000 \mathrm{~km}$ salinity gradient of the Baltic Sea. ISME J 5, 1571-1579. 
384 Hutchinson, G.E., 1961. The paradox of the plankton. American Naturalist, 137-145.

385 Jiang, X.-T., Peng, X., Deng, G.-H., Sheng, H.-F., Wang, Y., Zhou, H.-W., Tam, N.F.-Y., 2013. 386 Illumina sequencing of $16 \mathrm{~S}$ rRNA tag revealed spatial variations of bacterial communities in a 387 mangrove wetland. Microbial ecology 66, 96-104.

Kachel, V., Wietzorrek, J., 2000. Flow cytometry and integrated imaging. SCIENTIA MARINA $64,247-254$.

Kirkham, A.R., Lepere, C., Jardillier, L.E., Not, F., Bouman, H., Mead, A., Scanlan, D.J., 2013. A global perspective on marine photosynthetic picoeukaryote community structure. ISME J 7, $922-$ 936.

393

394

395

396

397

398

399

400

401

402

403

404

405

406

407

408

409

410

411

412

413

414

415

416

417

418

419

Kitamura, R., Ishii, K., Maeda, I., Kozaki, T., Iwabuchi, K., Saito, T., 2016. Evaluation of bacterial communities by bacteriome analysis targeting 16S rRNA genes and quantitative analysis of ammonia monooxygenase gene in different types of compost. Journal of bioscience and bioengineering 121, 57-65.

Le Bescot, N., Mahé, F., Audic, S., Dimier, C., Garet, M.J., Poulain, J., Wincker, P., Vargas, C., Siano, R., 2015. Global patterns of pelagic dinoflagellate diversity across protist size classes unveiled by metabarcoding. Environmental microbiology.

Logares, R., Sunagawa, S., Salazar, G., Cornejo-Castillo, F.M., Ferrera, I., Sarmento, H., Hingamp, P., Ogata, H., Vargas, C., Lima-Mendez, G., 2014. Metagenomic 16S rDNA Illumina tags are a powerful alternative to amplicon sequencing to explore diversity and structure of microbial communities. Environmental microbiology 16, 2659-2671.

Luo, C., Tsementzi, D., Kyrpides, N., Read, T., Konstantinidis, K.T., 2012. Direct Comparisons of Illumina vs. Roche 454 Sequencing Technologies on the Same Microbial Community DNA Sample. PLoS ONE 7, e30087.

Man-Aharonovich, D., Philosof, A., Kirkup, B.C., Le Gall, F., Yogev, T., Berman-Frank, I., Polz, M.F., Vaulot, D., Beja, O., 2010. Diversity of active marine picoeukaryotes in the Eastern Mediterranean Sea unveiled using photosystem-II psbA transcripts. ISME J 4, 1044-1052.

Mardis, E.R., 2008. Next-generation DNA sequencing methods. Annual review of genomics and human genetics 9, 387-402.

Massana, R., Gobet, A., Audic, S., Bass, D., Bittner, L., Boutte, C., Chambouvet, A., Christen, R., Claverie, J.M., Decelle, J., 2015. Marine protist diversity in European coastal waters and sediments as revealed by high-throughput sequencing. Environmental microbiology 17, 4035-4049.

McDonald, S.M., Sarno, D., Scanlan, D.J., Zingone, A., 2007. Genetic diversity of eukaryotic ultraphytoplankton in the Gulf of Naples during an annual cycle. Aquatic Microbial Ecology 50, 75-89.

Morán, X.A.G., LÓPEZ-URRUTIA, Á., CALVO-DÍAZ, A., Li, W.K., 2010. Increasing importance of small phytoplankton in a warmer ocean. Global Change Biology 16, 1137-1144. 
420 Olson, R.J., Shalapyonok, A., Sosik, H.M., 2003. An automated submersible flow cytometer for 421 analyzing pico-and nanophytoplankton: FlowCytobot. Deep Sea Research Part I: Oceanographic 422 Research Papers 50, 301-315.

423 Padilla, C.C., Ganesh, S., Gantt, S., Huhman, A., Parris, D.J., Sarode, N., Stewart, F.J., 2015. 424 Standard filtration practices may significantly distort planktonic microbial diversity estimates. 425 Frontiers in microbiology 6.

426 Parson, T., Maita, Y., Lalli, C., 1984. A Manual of Chemical and Biological Methods for Seawater 427 AnalysisPergamon Press. New York, 173.

428

429

430

431

432

433

434

435

436

437

438

439

440

441

442

443

444

445

446

447

448

449

450

451

452

453

454

455

Paul, J., Alfreider, A., Wawrik, B., 2000. Micro- and macrodiversity in rbcL sequences in ambient phytoplankton populations from the southeastern Gulf of Mexico Marine Ecology Progress Series 198, 9-18.

Pinto, A.J., Raskin, L., 2012. PCR Biases Distort Bacterial and Archaeal Community Structure in Pyrosequencing Datasets. PLoS ONE 7, e43093.

Schloss, P.D., Westcott, S.L., Ryabin, T., Hall, J.R., Hartmann, M., Hollister, E.B., Lesniewski, R.A., Oakley, B.B., Parks, D.H., Robinson, C.J., Sahl, J.W., Stres, B., Thallinger, G.G., Van Horn, D.J., Weber, C.F., 2009. Introducing mothur: open-source, platform-independent, communitysupported software for describing and comparing microbial communities. Applied and environmental microbiology 75, 7537-7541.

Schmidt, P.-A., Bálint, M., Greshake, B., Bandow, C., Römbke, J., Schmitt, I., 2013. Illumina metabarcoding of a soil fungal community. Soil Biology and Biochemistry 65, 128-132.

Shakya, M., Quince, C., Campbell, J.H., Yang, Z.K., Schadt, C.W., Podar, M., 2013. Comparative metagenomic and rRNA microbial diversity characterization using archaeal and bacterial synthetic communities. Environmental microbiology 15, 1882-1899.

Sherwood, A.R., Presting, G.G., 2007. Universal Primers Amplify a 23s Rdna Plastid Marker in Eukaryotic Algae and Cyanobacteria1. Journal of phycology 43, 605-608.

Staley, C., Unno, T., Gould, T., Jarvis, B., Phillips, J., Cotner, J., Sadowsky, M., 2013. Application of Illumina next-generation sequencing to characterize the bacterial community of the Upper Mississippi River. Journal of applied microbiology 115, 1147-1158.

Steven, B., McCann, S., Ward, N.L., 2012. Pyrosequencing of plastid 23S rRNA genes reveals diverse and dynamic cyanobacterial and algal populations in two eutrophic lakes. FEMS microbiology ecology 82, 607-615.

Valenzuela-González, F., Martínez-Porchas, M., Villalpando-Canchola, E., Vargas-Albores, F., 2016. Studying long $16 \mathrm{~S}$ rDNA sequences with ultrafast-metagenomic sequence classification using exact alignments (Kraken). Journal of Microbiological Methods.

Vierheilig, J., Savio, D., Ley, R., Mach, R., Farnleitner, A., Reischer, G., 2015. Potential applications of next generation DNA sequencing of 16S rRNA gene amplicons in microbial water 
456 quality monitoring. Water Science and Technology 72, 1962-1972.

457 Zeidner, G., Preston, C.M., Delong, E.F., Massana, R., Post, A.F., Scanlan, D.J., Béjà, O., 2003.

458 Molecular diversity among marine picophytoplankton as revealed by psbA analyses. 459 Environmental Microbiology 5, 212-216.

460

461

462

463

464

465

466

467

468

469

470

471

472

473

474

475

476

477

478

479

480

481

482 
Fig. 1. Workflow of PHYTOGEN database process

488

489

490

491

492

493

494

495

496

497

498

499

500

501

502

503
Fig. 2. Composition of phytoplankton by the different cut-off similarity of OTUs

(A) Each bar shows the ratio of phytoplankton phyla according to cut-off similarity from $90 \%$ to $99 \%$. (B) Each bar shows the ratio of phytoplankton classes according to cut-off similarity from $90 \%$ to $99 \%$.

Fig. 3. Coverage of obtained OTUs in algal phyla

Phylogenetic tree was constructed by the Neigbhor-joining (NJ) algorithm using Molecular Evolutionary Genetics Analysis (MEGA ver 6.0). The evolutionary distances were computed using the Kimura 2-parameter method.

Fig. 4. (A) Ratio of algal phyla in each sample with different locations, depths, and seasons Each bar shows the ratio of phytoplankton phyla according to $97 \%$ cut-off similarity (B)Venn diagram of OTUs for the four surface samples in East/Japan Sea The venn diagram was generated with Draw Venn Diagram (http://bioinformatics.psb. ugent.be/webtools/Venn/) 
504

505

506

507

508

509

510
Fig. 1.

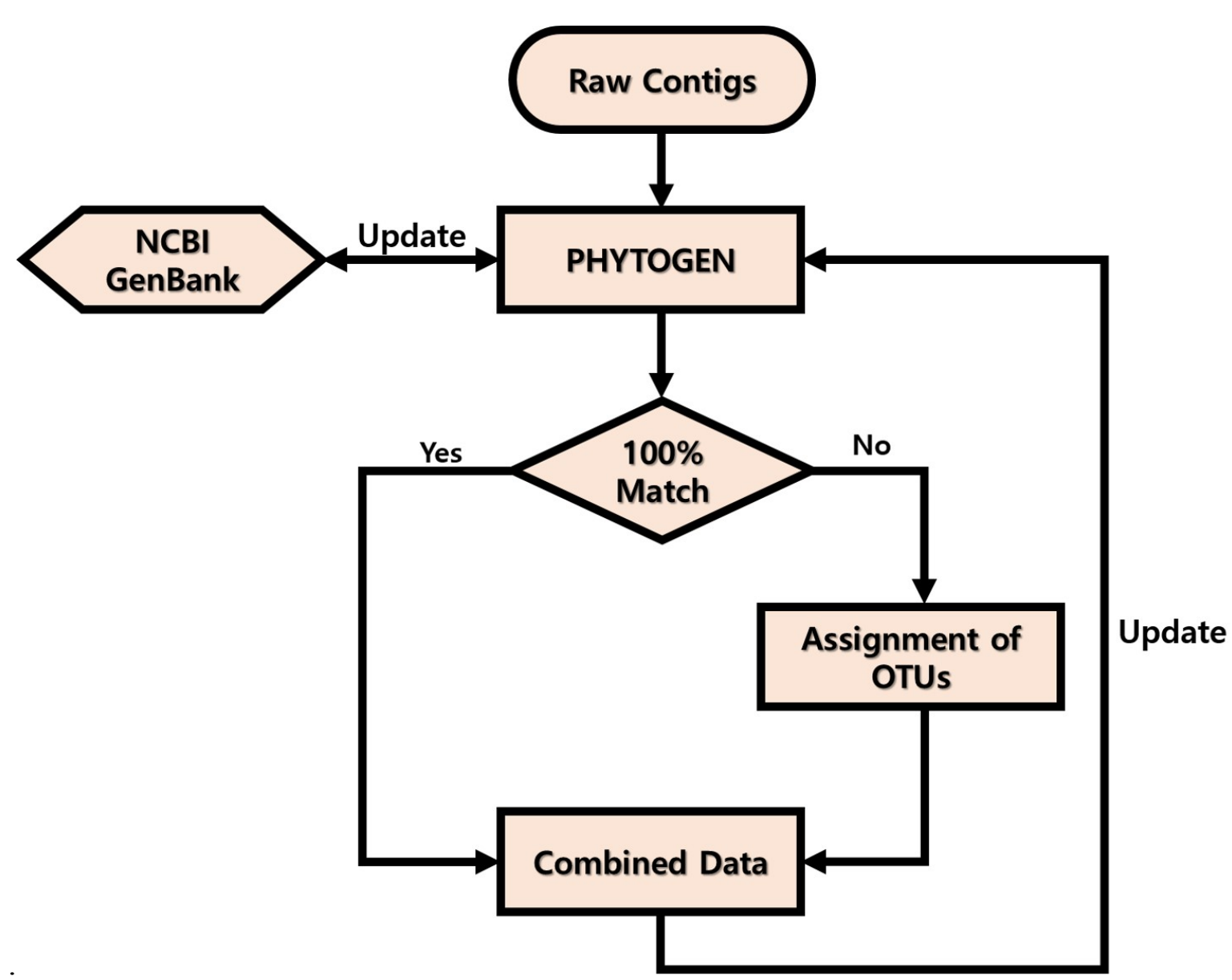


511 Fig. 2. 512 (A)

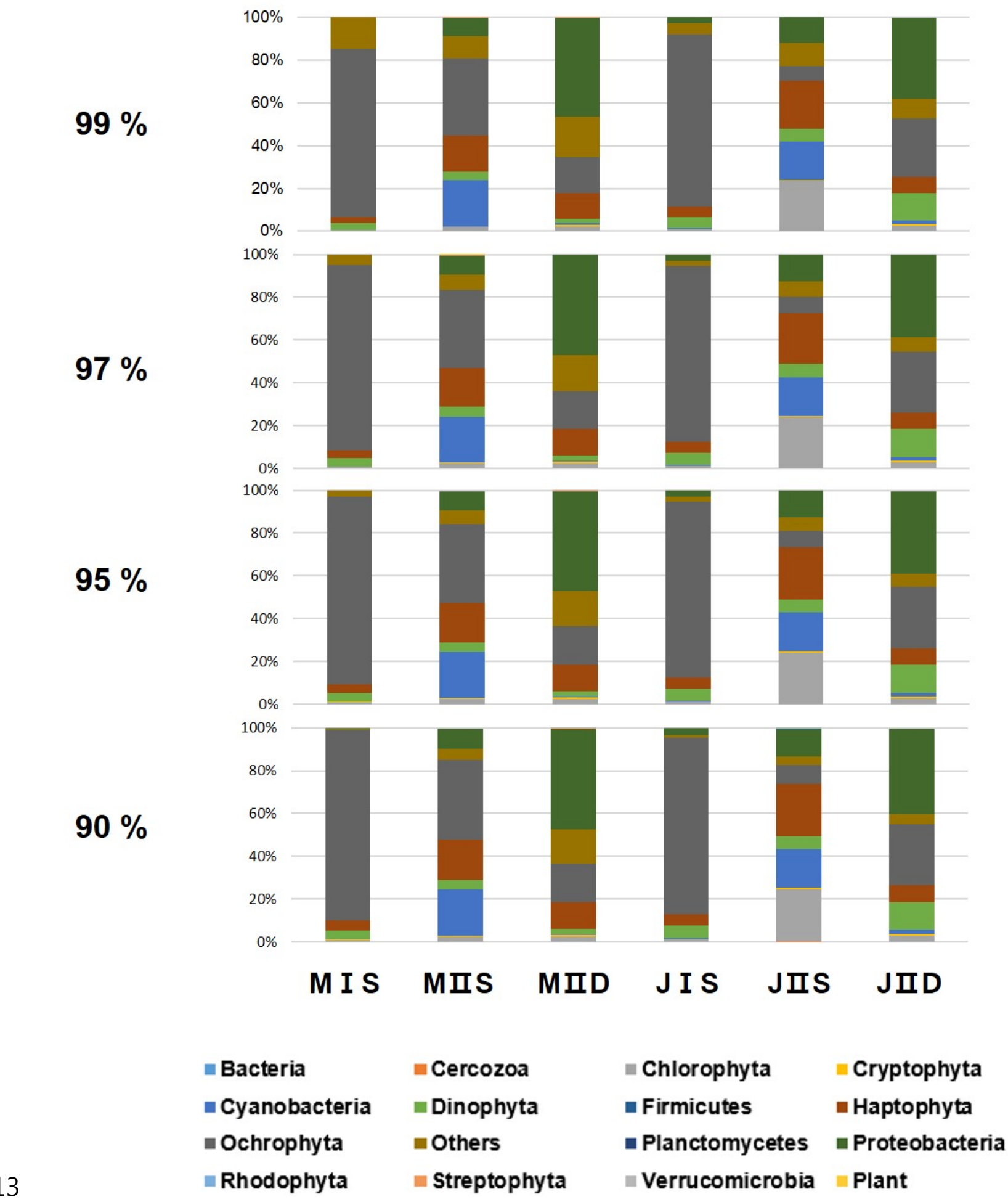


$100 \%$
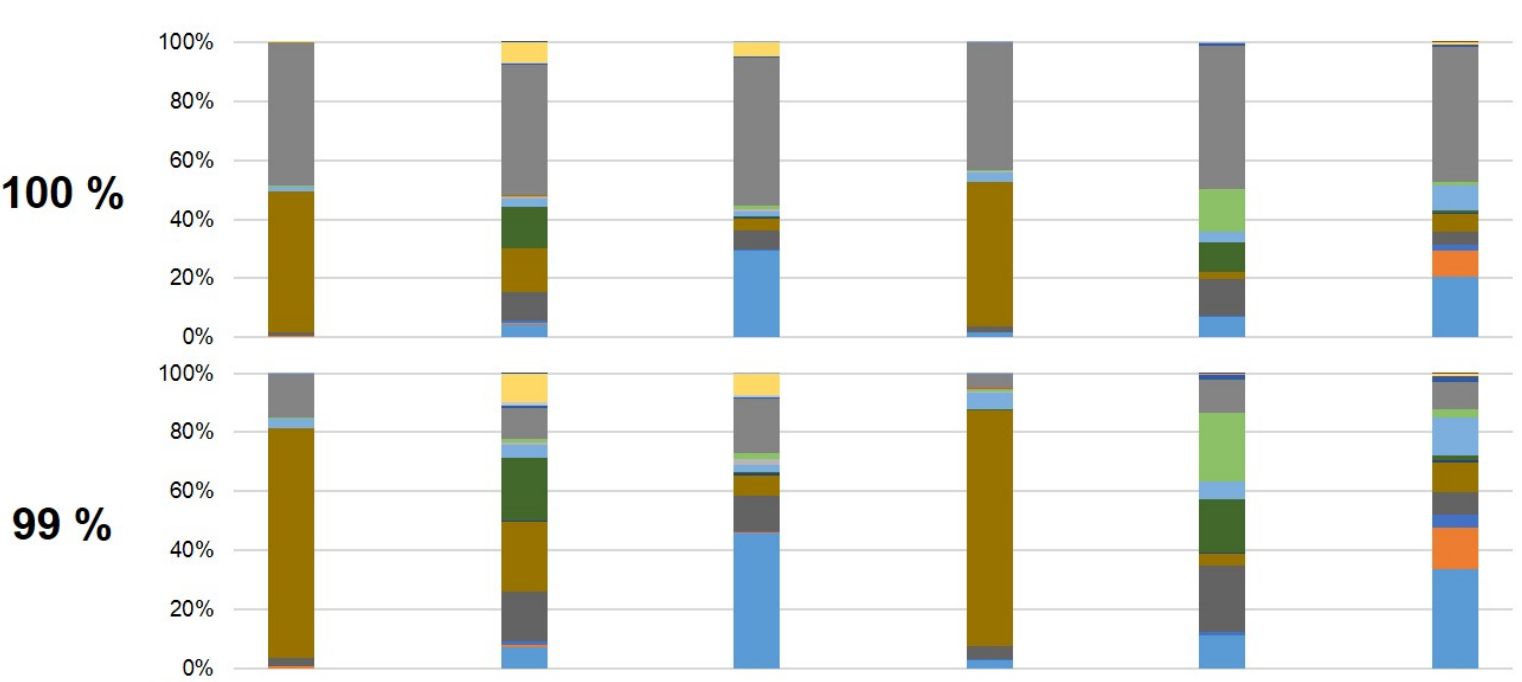

\section{$98 \%$}
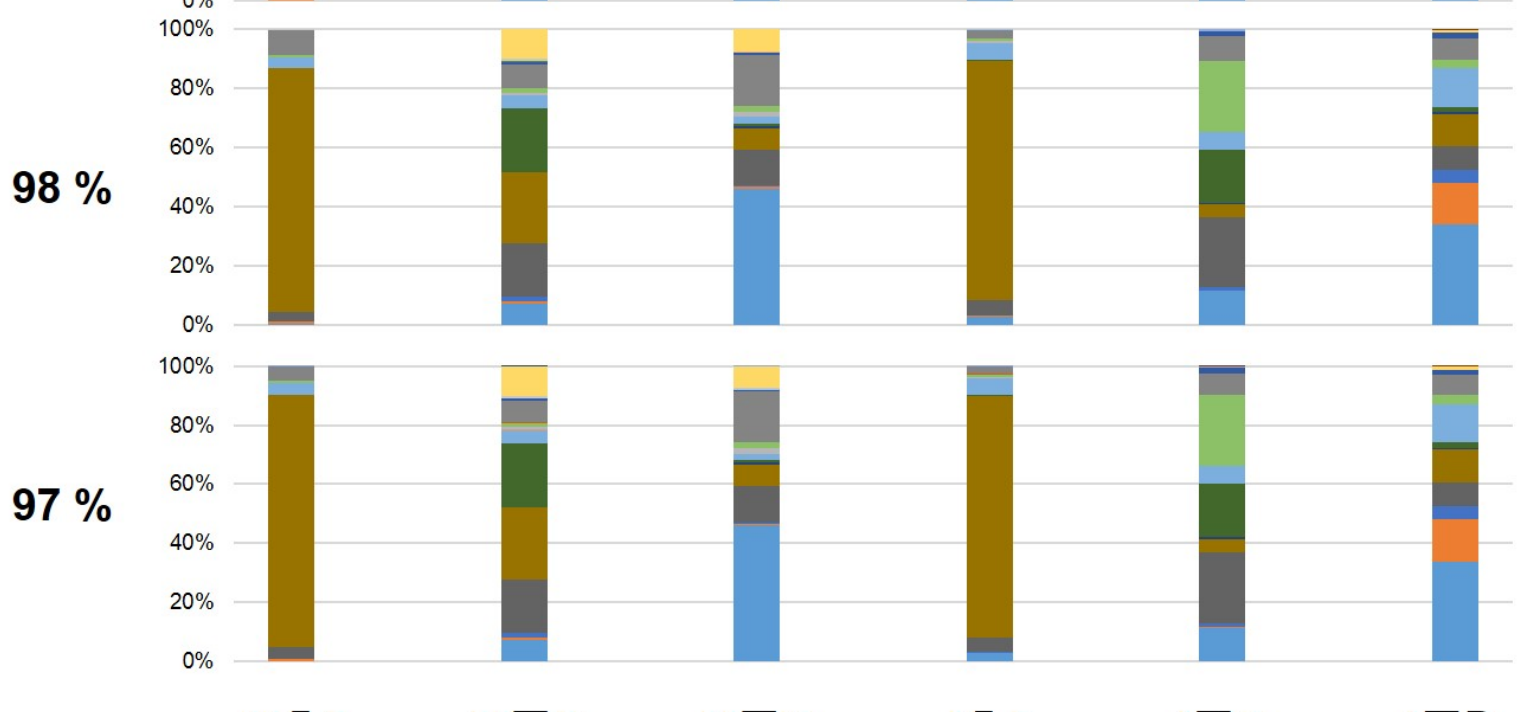

\section{I S MIIS MIID}

J I S

JIIS

JIID

- Alphaproteobacteria
- Betaproteobacteria
- Coccolithophyceae
- Dinophyceae
" Liliopsida
- Others
" Pinopsida
- Synurophyceae

Bacillariophyceae

Chlorodendrophyceae

- Coscinodiscophyceae

- Florideophyceae

n Mamiellophyceae

- Pavlovophyceae

- Prasinophyceae

- Trebouxiophyceae

- Bacilli
- Chlorophyceae
- Cryptophyceae
- Fragilariophyceae
- Negativicutes
- Pelagophyceae
- Pyramimonadophyceae
- Ulvophyceae

Bangiophyceae

- Clostridia

- Cyanophyceae

- Gammaproteobacteria

- Opitutae

- Phaeophyceae

Raphidophyceae

- Verrucomicrobiae 


\section{Fig. 3.}

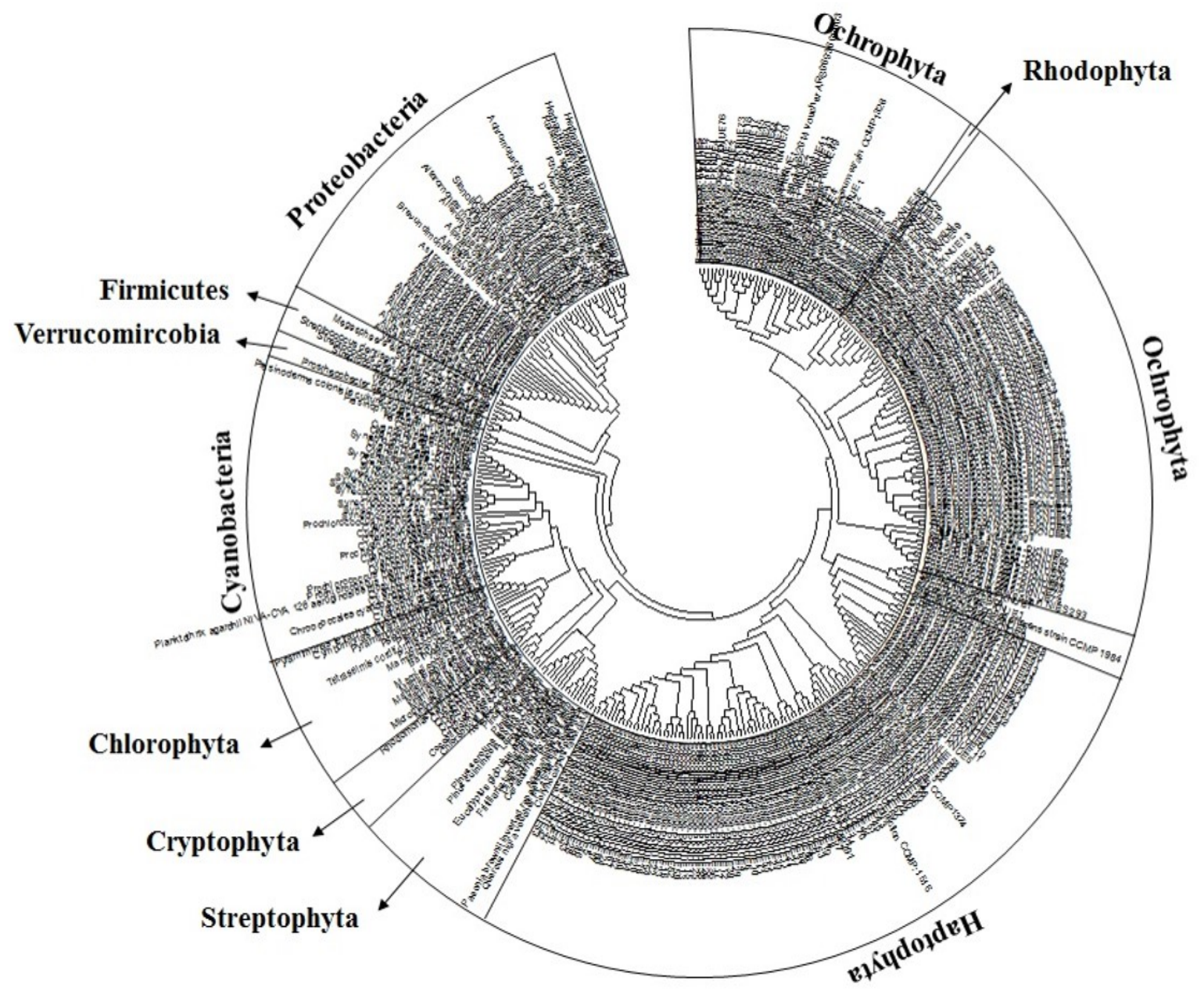

522

523 
524 Fig. 4.

525

$526 \quad$ (A)

527

528

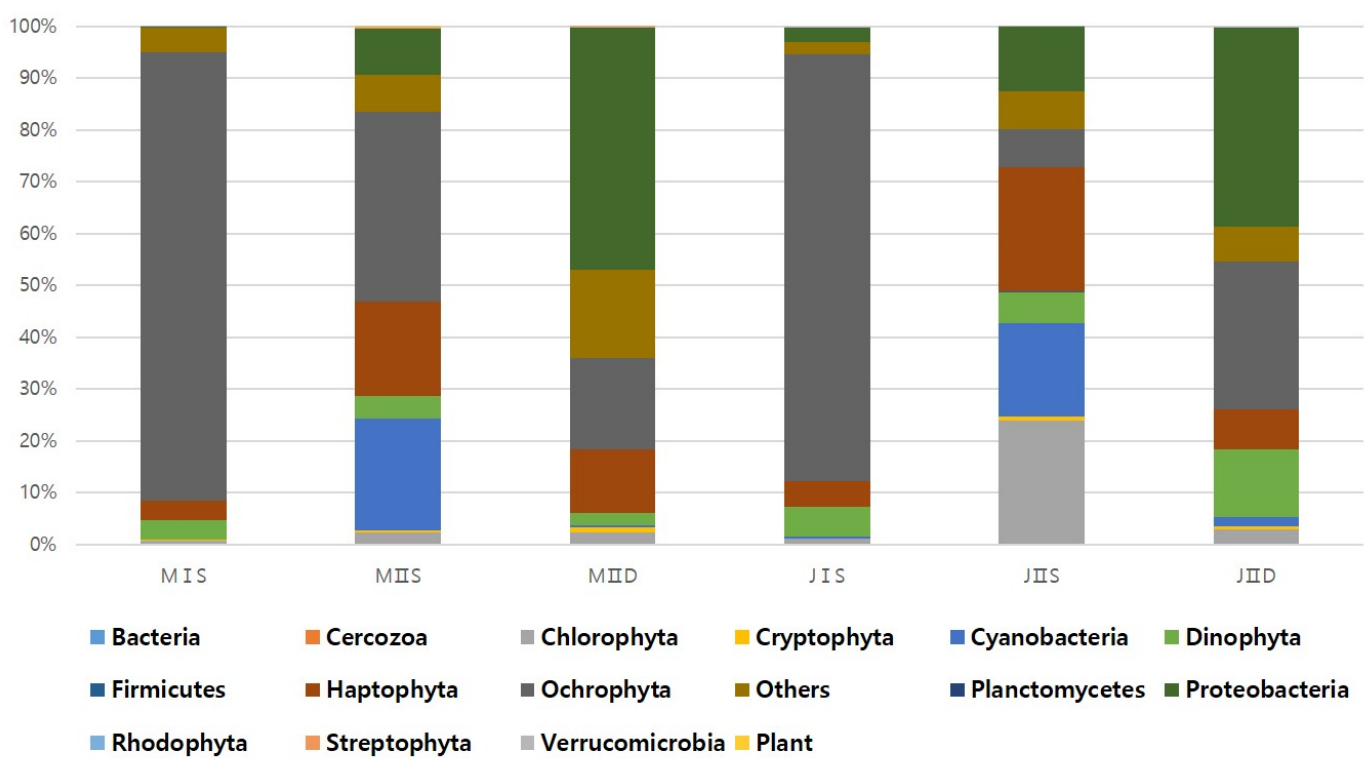

529

$530 \quad$ (B)

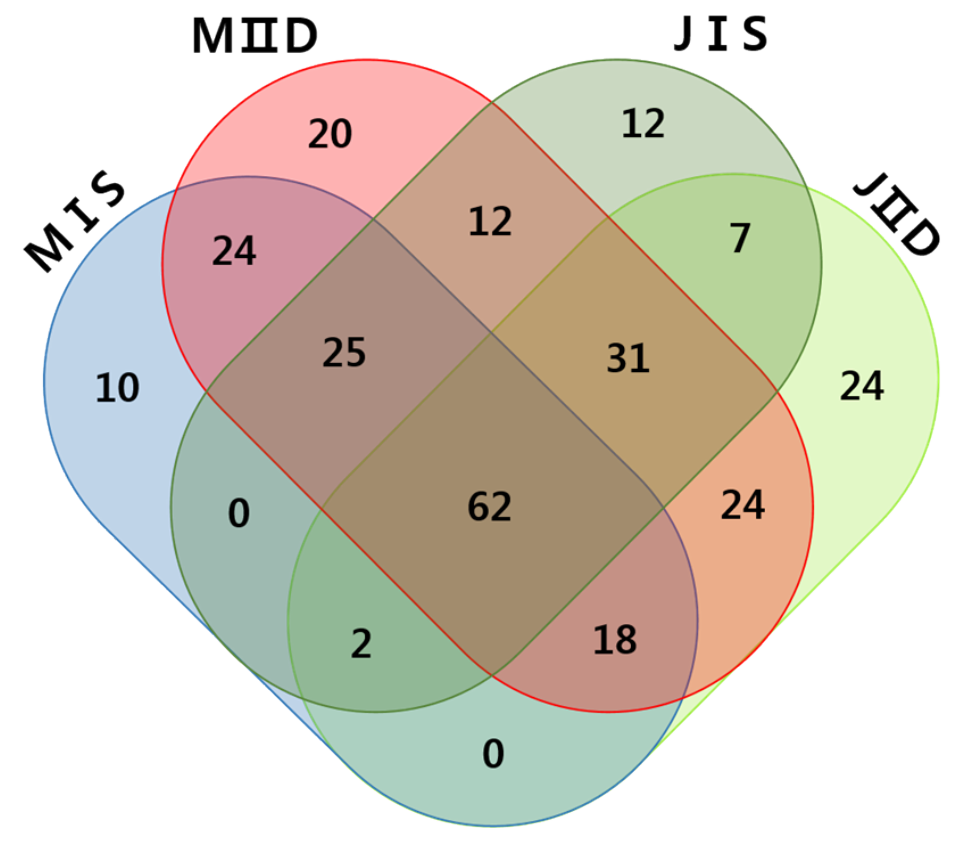


534 Table 1 Consensus Nucleotide sequences of forward primer targeting 23S rDNA region

\begin{tabular}{|c|c|c|c|c|c|c|c|c|c|c|c|c|c|c|c|c|c|c|c|c|c|c|c|c|c|}
\hline A23SrVF1 & & $5^{\prime}$ & G & G & A & c & A & $R$ & A & A & A & G & A & c & c & c & $\mathrm{T}$ & A & $\mathrm{T}$ & G & & & & & \\
\hline A23SrVR1 & & & & & & c & A & $R$ & A & A & A & G & A & c & c & c & $\mathrm{T}$ & A & $T$ & G & M & A & G & C & $T$ \\
\hline $\begin{array}{l}\text { P23Rv f1 } \\
\text { (Sherwood and Presting, 2007) }\end{array}$ & & $5^{\prime}$ & G & G & A & c & A & G & A & A & A & G & A & c & c & c & T & A & T & G & A & A & - & - & - \\
\hline Concensus sequence(Algae) & & & G & G & A & c & A & $\mathrm{R}$ & A & A & A & G & $\mathrm{R}$ & c & c & $\mathrm{Y}$ & Y & A & T & G & $\mathrm{M}$ & A & $\mathrm{s}$ & c & $T$ \\
\hline Phylum & Total & & & & & & & & & & & & & & & & & & & & & & & & \\
\hline \multirow{2}{*}{ Chlorophyta } & \multirow{2}{*}{113} & & G & $\mathrm{G}$ & A & c & A & A & A & A & A & G & A & c & c & c & T & A & T & G & A & A & G & c & T \\
\hline & & & 113 & 113 & 113 & 113 & 113 & 113 & 113 & 113 & 113 & 113 & 113 & 113 & 113 & 113 & 113 & 113 & 113 & 113 & 113 & 113 & 113 & 113 & 113 \\
\hline \multirow{2}{*}{ Cryptophyta } & \multirow{2}{*}{\multicolumn{2}{|c|}{54}} & G & G & A & c & A & G & A & A & A & G & A & c & c & c & $T$ & A & $T$ & G & A & A & G & c & $T$ \\
\hline & & & 54 & 54 & 54 & 54 & 54 & 54 & 54 & 54 & 54 & 54 & 54 & 54 & 54 & 54 & 54 & 54 & 54 & 54 & 54 & 54 & 54 & 54 & 54 \\
\hline \multirow{2}{*}{ Euglenophyta } & \multirow{2}{*}{\multicolumn{2}{|c|}{141}} & G & G & A & c & A & G & A & A & A & G & A & c & c & $Y(C / T)$ & $T$ & A & $T$ & G & $M(A / C)$ & A & G & c & $\mathrm{T}$ \\
\hline & & & 141 & 141 & 141 & 141 & 141 & 141 & 141 & 141 & 141 & 141 & 141 & 141 & 141 & $140 / 1$ & 141 & 141 & 141 & 141 & $137 / 4$ & 141 & 141 & 141 & 141 \\
\hline \multirow{2}{*}{ Ochrophyta } & \multirow{2}{*}{\multicolumn{2}{|c|}{26}} & G & G & A & c & A & G & A & A & A & G & A & c & c & c & $T$ & A & $\mathrm{T}$ & G & A & A & G & c & $T$ \\
\hline & & & 26 & 26 & 26 & 26 & 26 & 26 & 26 & 26 & 26 & 26 & 26 & 26 & 26 & 26 & 26 & 26 & 26 & 26 & 26 & 26 & 26 & 26 & 26 \\
\hline \multirow{2}{*}{ Streptophyta } & \multirow{2}{*}{\multicolumn{2}{|c|}{198}} & G & G & A & $c$ & A & G & A & A & A & G & A & c & $c$ & $c$ & $T$ & A & $T$ & G & A & A & G & $c$ & $\mathrm{~T}$ \\
\hline & & & 198 & 198 & 198 & 198 & 198 & 198 & 198 & 198 & 198 & 198 & 198 & 198 & 198 & 198 & 198 & 198 & 198 & 198 & 198 & 198 & 198 & 198 & 198 \\
\hline \multirow{2}{*}{ Dinophyta } & \multirow{2}{*}{\multicolumn{2}{|c|}{13}} & G & G & A & c & A & $R(A / G)$ & A & A & A & G & $R(A / G)$ & c & c & c & $T$ & A & $T$ & G & A & A & $s$ & c & $T$ \\
\hline & & & 13 & 13 & 13 & 13 & 13 & $1 / 12$ & 13 & 13 & 13 & 13 & $12 / 1$ & 13 & 13 & 13 & 13 & 13 & 13 & 13 & 13 & 13 & $12 / 1$ & 13 & 13 \\
\hline \multirow{2}{*}{ Cyanobacteria } & \multirow{2}{*}{\multicolumn{2}{|c|}{195}} & G & G & A & c & A & G & A & A & A & G & A & c & c & c & $Y(C / T)$ & A & $T$ & G & A & A & G & c & $\mathrm{T}$ \\
\hline & & & 195 & 195 & 195 & 195 & 195 & 195 & 195 & 195 & 195 & 195 & 195 & 195 & 195 & 195 & $194 / 1$ & 195 & 195 & 195 & 195 & 195 & 195 & 195 & 195 \\
\hline \multirow{2}{*}{ Proteobacteria- $\alpha$} & \multirow{2}{*}{\multicolumn{2}{|c|}{198}} & * & G & A & c & $R(A / G)$ & G & A & A & A & $R(A / G)$ & A & c & c & c & $Y(C / T)$ & $\mathrm{R}$ & $T$ & G & $M(A / C)$ & A & c & c & $T$ \\
\hline & & & ${ }^{*}$ & 198 & 198 & 198 & 1/197 & 198 & 198 & 198 & 198 & 2/196 & 198 & 198 & 198 & 198 & 96/102 & 109/89 & 198 & 198 & 98/100 & 198 & 198 & 198 & 198 \\
\hline \multirow{2}{*}{ Proteobacteria- $\beta$} & \multirow{2}{*}{\multicolumn{2}{|c|}{198}} & * & G & A & c & G & G & A & A & A & G & A & c & c & c & $T$ & A & $T$ & G & A & A & $c$ & c & $T$ \\
\hline & & & & 198 & 198 & 198 & 198 & 198 & 198 & 198 & 198 & 198 & 198 & 198 & 198 & 198 & 198 & 198 & 198 & 198 & 198 & 198 & 198 & 198 & 198 \\
\hline \multirow{2}{*}{ Proteobacteria- $\gamma$} & \multirow{2}{*}{\multicolumn{2}{|c|}{198}} & * & G & A & c & G & G & A & A & A & G & A & c & c & c & $T$ & $\mathrm{R}$ & $\mathrm{T}$ & G & $M(A / C)$ & A & c & c & $T$ \\
\hline & & & 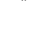 & 198 & 198 & 198 & 198 & 198 & 198 & 198 & 198 & 198 & 198 & 198 & 198 & 198 & 198 & $1 / 197$ & 198 & 198 & $181 / 17$ & 198 & 198 & 198 & 198 \\
\hline \multirow[t]{2}{*}{ 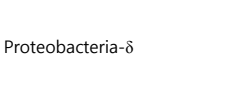 } & \multirow{2}{*}{\multicolumn{2}{|c|}{151}} & * & G & $R(A / G)$ & $M(C / A)$ & $R(A / G)$ & G & $R(A / G)$ & A & A & $R(A / G)$ & R & $M(A / C)$ & c & c & $Y(C / T)$ & $\begin{array}{c}\mathrm{N} \\
(\mathrm{A} / \mathrm{G} / \mathrm{C} / \mathrm{T})\end{array}$ & $\mathrm{K}(\mathrm{G} / \mathrm{T})$ & $\begin{array}{c}D \\
(A / G / T)\end{array}$ & $\begin{array}{c}V \\
(A / G / C)\end{array}$ & $W(A / T)$ & $M(A / C)$ & $Y(C / T)$ & $Y(C / T)$ \\
\hline & & & & 151 & $147 / 4$ & $147 / 4$ & $21 / 126$ & 151 & $147 / 4$ & 151 & 151 & 4/147 & $147 / 4$ & $4 / 147$ & 151 & 151 & $32 / 119$ & $15 / 132 / 2 / 2$ & 9/142 & $5 / 142 / 4$ & $90 / 7 / 54$ & $143 / 8$ & $4 / 147$ & $146 / 5$ & 4/147 \\
\hline & & & * & G & A & G & G & G & $R(A / G)$ & $R(A / G)$ & A & G & A & c & c & c & $T$ & G & $T$ & G & $s(C / G)$ & A & c & c & $\mathrm{T}$ \\
\hline Proteobacteria- $\varepsilon$ & 198 & & * & 198 & 198 & 198 & 198 & 198 & $196 / 2$ & $190 / 8$ & 198 & 198 & 198 & 198 & 198 & 198 & 198 & 198 & 198 & 198 & $150 / 48$ & 198 & 198 & 198 & 198 \\
\hline
\end{tabular}


537 Table 2. Comparison of phytoplankton sequences between the modified primer and original universal primer

\begin{tabular}{|c|c|c|c|c|c|c|}
\hline \multirow{2}{*}{ Phylum } & \multicolumn{3}{|c|}{ Modified Primers } & \multicolumn{3}{|c|}{ Original Primers } \\
\hline & Amplicons & $\%$ & OTUs & Amplicons & $\%$ & OTUs \\
\hline Ochrophyta & 398,588 & 63.68 & 76 & 24,006 & 12.92 & 31 \\
\hline Unknown & 122,178 & 19.52 & - & 81,562 & 43.90 & - \\
\hline Haptophyta & 47,816 & 7.64 & 22 & 2,198 & 1.18 & 6 \\
\hline Dinophyta & 28,008 & 4.47 & 5 & 1,510 & 0.81 & 3 \\
\hline Chlorophyta & 828 & 0.13 & 1 & 226 & 0.12 & 1 \\
\hline Total & 625,917 & 100 & 106 & 185,773 & 100 & 63 \\
\hline
\end{tabular}


541 Table 3. Summary of end-paired phytoplanktonic contigs and processed OTUs in samples of East/Japan Sea 542

\begin{tabular}{|c|c|c|c|c|c|c|c|c|c|c|c|c|c|c|c|c|c|c|}
\hline \multirow{2}{*}{ Phylum } & \multicolumn{3}{|c|}{ M I S } & \multicolumn{3}{|c|}{ M II S } & \multicolumn{3}{|c|}{ M II D } & \multicolumn{3}{|c|}{ J I S } & \multicolumn{3}{|c|}{$\mathbf{J}$ II S } & \multicolumn{3}{|c|}{ J II D } \\
\hline & Contigs & $\begin{array}{l}\text { Ratio } \\
((\%) \\
\end{array}$ & OTUs & Contigs & $\begin{array}{c}\text { Ratio } \\
(\%)\end{array}$ & OTUs & Contigs & $\begin{array}{c}\text { Ratio } \\
(\%)\end{array}$ & OTUs & Contigs & $\begin{array}{c}\text { Ratio } \\
(\%)\end{array}$ & OTUs & Contigs & $\begin{array}{c}\text { Ratio } \\
(\%)\end{array}$ & OTUs & Contigs & $\begin{array}{c}\text { Ratio } \\
(\%)\end{array}$ & OTUs \\
\hline Chlorophyta & 4,815 & 0.75 & 21 & 7,261 & 2.30 & 22 & 8,656 & 2.26 & 15 & 7,036 & 1.13 & 13 & 98,420 & 23.91 & 19 & 8,279 & 2.85 & 14 \\
\hline Cryptophyta & 1,063 & 0.17 & 6 & 1,151 & 0.36 & 5 & 3,684 & 0.96 & 4 & 24 & 0.00 & 4 & 2,839 & 0.69 & 4 & 1,878 & 0.65 & 5 \\
\hline Cyanobacteria & 209 & 0.03 & 17 & 67,933 & 21.54 & 30 & 2,029 & 0.53 & 19 & 2,561 & 0.41 & 15 & 74,420 & 18.08 & 31 & 5,264 & 1.81 & 26 \\
\hline Dinophyta & 24,441 & 3.82 & 19 & 14,173 & 4.49 & 16 & 8,919 & 2.33 & 15 & 35,103 & 5.65 & 14 & 24,917 & 6.05 & 16 & 37,744 & 13.01 & 17 \\
\hline Firmicutes & - & 0.00 & 0 & 2 & 0.00 & 2 & - & 0.00 & 0 & - & 0.00 & 0 & 38 & 0.01 & 2 & 123 & 0.04 & 2 \\
\hline Haptophyta & 24,094 & 3.77 & 74 & 57,385 & 18.20 & 75 & 46,724 & 12.22 & 73 & 31,230 & 5.03 & 70 & 98,841 & 24.01 & 76 & 22,534 & 7.77 & 75 \\
\hline Ochrophyta & 552,850 & 86.40 & 118 & 115,611 & 36.66 & 119 & 67,856 & 17.74 & 113 & 510,993 & 82.31 & 109 & 30,117 & 7.32 & 118 & 82,463 & 28.43 & 123 \\
\hline Unclassified & 30,246 & 4.73 & $\mathrm{~N} / \mathrm{A}$ & 22,399 & 7.10 & $\mathrm{~N} / \mathrm{A}$ & 65,121 & 17.03 & $\mathrm{~N} / \mathrm{A}$ & 14,556 & 2.34 & $\mathrm{~N} / \mathrm{A}$ & 30,478 & 7.40 & $\mathrm{~N} / \mathrm{A}$ & 19,497 & 6.72 & N/A \\
\hline Proteobacteria & 1,177 & 0.18 & 19 & 27,885 & 8.84 & 46 & 178,140 & 46.57 & 41 & 17,715 & 2.85 & 32 & 50,790 & 12.34 & 46 & 111,346 & 38.38 & 54 \\
\hline Rhodophyta & 772 & 0.12 & 1 & 315 & 0.10 & 1 & 16 & 0.00 & 1 & 2 & 0.00 & 1 & 22 & 0.01 & 1 & 28 & 0.01 & 1 \\
\hline Streptophyta & 52 & 0.01 & 4 & 424 & 0.13 & 4 & 1,103 & 0.29 & 8 & 422 & 0.07 & 6 & 404 & 0.10 & 9 & 7 & 0.00 & 3 \\
\hline Verrucomicrobia & 117 & 0.02 & 2 & 807 & 0.26 & 2 & 253 & 0.07 & 2 & 1,200 & 0.19 & 2 & 312 & 0.08 & 3 & 923 & 0.32 & 4 \\
\hline Plant & - & 0.00 & 0 & 1 & 0.00 & 1 & - & 0.00 & 0 & - & 0.00 & 0 & - & 0.00 & 0 & - & 0.00 & 0 \\
\hline Total & 639,836 & 100 & 281 & 315,347 & 100 & 323 & 382,501 & 100 & 291 & 620,842 & 100 & 266 & 411,598 & 100 & 325 & 290,086 & 100 & 324 \\
\hline
\end{tabular}


545 Table 4. Top 20 phytoplanktonic OTUs in each sample of East/Japan Sea

546

\begin{tabular}{|c|c|c|c|c|c|c|c|c|c|c|c|}
\hline MIS & & MIIS & & MID & & JIS & & $\mathrm{JIIS}$ & & $\mathrm{JIID}$ & \\
\hline OTUs & $\%$ & отUs & $\%$ & OTUs & $\%$ & OTUs & $\%$ & отUs & $\%$ & отUs & $\%$ \\
\hline Coscinodiscophyceae-PKNUE1 & 58.34 & Cyanophyceae-PKNUE1 & 14.21 & Heterosigma akashiwo - EU168190 & 7.47 & Coscinodiscophyceaa-PKNUE52 & 23.33 & Mamiellophyceae-PKNUE1 & 7.06 & Bacillariophycea-PKNUE4 & 12.93 \\
\hline Coscinodiscophycea-PKNUE2 & 2.63 & Coscinodiscophyceae-PKNUE1 & 11.79 & Coccolithophyceae-PKNUE71 & 3.20 & Coscinodiscophyceae-PKNUE55 & 16.61 & Synechococcus sp. - CP000110 & 6.48 & Dinophyceae-PKNUE10 & 10.89 \\
\hline Coscinodiscophyceae-PKNUE79 & 2.56 & Heterosigma akashiwo - EU168190 & 9.91 & Fragilariophyceae-PKNUE1 & 1.90 & Coscinodiscophyceae-PKNUE25 & 9.04 & Dinophyceae-PKNUE10 & 3.84 & Coccolithophyceae-PKNUE4 & 2.49 \\
\hline Dinophyceae-PKNUE7 & 1.63 & Synechococcus sp. - CP000097 & 5.04 & Phaeocystis antarctica - JN117275 & 1.24 & Coscinodiscophyceae-PKNUE56 & 4.67 & Coccolithophyceae-PKNUE4 & 3.59 & Mamiellophyceae-PKNUE8 & 1.58 \\
\hline Coscinodiscophyceae-PKNUE49 & 0.99 & Coccolithophyceae-PKNUE42 & 1.94 & Coscinodiscophyceae-PKNUE92 & 0.89 & Coscinodiscophyceae-PKNUE57 & 3.69 & Micromonas pusilla - FN563097 & 3.37 & Coscinodiscophyceae-PKNUE55 & 1.46 \\
\hline Coscinodiscophyceae-PKNUE41 & 0.89 & Dinophyceae-PKNUE10 & 1.93 & Coscinodiscophyceae-PKNUE1 & 0.83 & Coscinodiscophyceae-PKNUE58 & 2.18 & Prochlorococcus marinus - CP000576 & 3.34 & Coscinodiscophyceae-PKNUE74 & 1.34 \\
\hline Coscinodiscophyceae-PKNUE4 & 0.87 & Coccolithophyceae-PKNUE5 & 1.81 & Coscinodiscophyceae-PKNUE51 & 0.73 & Dinophycea-PKNUE4 & 2.07 & Mamiellophyceae-PKNUE3 & 3.04 & Coscinodiscophycea-PKNUE90 & 1.22 \\
\hline Coscinodiscophyceae-PKNUE3 & 0.76 & Coccolithophyceae-PKNUE6 & 1.08 & Cryptophyceae-PKNUE1 & 0.69 & Coscinodiscophyceae-PKNUE59 & 1.87 & Mamiellophyccae-PKNUE6 & 3.02 & Dinophyceae-PKNUE6 & 1.21 \\
\hline Coscinodiscophyceae-PKNUE40 & 0.72 & Coscinodiscophyceae-PKNUE13 & 1.06 & Coccolithophyceae-PKNUE50 & 0.68 & Dinophyceae-PKNUE10 & 1.80 & Synechococcus sp. - CP006882 & 2.40 & Coccolithophyceae-PKNUE38 & 1.18 \\
\hline Coscinodiscophyceae-PKNUE19 & 0.65 & Dinophyceae-PKNUE7 & 1.04 & Dinophyceae-PKNUE11 & 0.64 & Coscinodiscophycea-PKNUE60 & 1.67 & Mamiellophyceae-PKNUE2 & 2.18 & Mamiellophyceae-PKNUE1 & 1.09 \\
\hline Coscinodiscophyceae-PKNUE91 & 0.62 & Coscinodiscophyceae-PKNUE2 & 1.02 & Mamiellophyceae-PKNUE6 & 0.54 & Coscinodiscophyceae-PKNUE26 & 1.29 & Mamiellophyceae-PKNUE4 & 1.87 & Coccolithophyceae-PKNUE7 & 1.03 \\
\hline Coscinodiscophyceae-PKNUE5 & 0.58 & Coscinodiscophyceae-PKNUE90 & 0.98 & Mamiellophycea-PKNUE8 & 0.50 & Dinophyceae-PKNUE7 & 1.05 & Mamiellophyceae-PKNUE8 & 1.78 & Coscinodiscophycea-PKNUE18 & 1.01 \\
\hline Coscinodiscophyceae-PKNUE6 & 0.51 & Coccolithophyceae-PKNUE40 & 0.91 & Coccolithophyceae-PKNUE73 & 0.49 & Coscinodiscophyceae-PKNUE27 & 0.98 & Coccolithophyceae-PKNUE50 & 1.69 & Coscinodiscophyceae-PKNUE52 & 0.74 \\
\hline Coscinodiscophyceae-PKNUE80 & 0.50 & Coscinodiscophyceae-PKNUE51 & 0.84 & Coccolithophyceae-PKNUE47 & 0.49 & Coscinodiscophyceae-PKNUE82 & 0.93 & Dinophyceae-PKNUE12 & 1.62 & Coscinodiscophyceae-PKNUE76 & 0.73 \\
\hline Dinophyceae-PKNUE8 & 0.45 & Coccolithophyceae-PKNUE43 & 0.82 & Coccolithophyceae-PKNUE4 & 0.47 & Coscinodiscophyceae-PKNUE28 & 0.83 & Synechococcus sp. - CP000097 & 1.45 & Coscinodiscophycea-PKNUE89 & 0.71 \\
\hline Coscinodiscophyceae-PKNUE8 & 0.43 & Mamiellophycea-PKNUE8 & 0.77 & Coscinodiscophyceae-PKNUE24 & 0.47 & Coscinodiscophyceae-PKNUE1 & 0.67 & Coccolithophyceae-PKNUE21 & 1.25 & Coscinodiscophyceae-PKNUE75 & 0.69 \\
\hline Bacillariophyceac-PKNUE2 & 0.42 & Coccolithophycea-PKNUE1 & 0.76 & Mamiellophyceae-PKNUE1 & 0.46 & Coscinodiscophyceaa-PKNUE45 & 0.66 & Coscinodiscophyceae-PKNUE94 & 1.23 & Cyanophycaa-PKNUE12 & 0.68 \\
\hline Coscinodiscophyceae-PKNUE21 & 0.40 & Coccolithophycaa-PKNUE44 & 0.72 & Coscinodiscophyceae-PKNUE52 & 0.46 & Coscinodiscophyceaa-PKNUE61 & 0.59 & Mamiellophyceae-PKNUE5 & 1.09 & Coccolithophyceae-PKNUE64 & 0.66 \\
\hline Coscinodiscophyceae-PKNUE48 & 0.40 & Fragilariophyccae-PKNUE1 & 0.68 & Coscinodiscophyceae-PKNUE20 & 0.43 & Coscinodiscophyceae-PKNUE62 & 0.55 & Coccolithophyceae-PKNUE54 & 0.84 & Coccolithophyceae-PKNUE46 & 0.65 \\
\hline Dinophyceae-PKNUE1 & 0.40 & Coscinodiscophyceae-PKNUE92 & 0.68 & Coccolithophyceae-PKNUE15 & 0.42 & Coscinodiscophyceae-PKNUE63 & 0.54 & Cyanophyceae-PKNUE5 & 0.69 & Bacillariophyceae-PKNUE5 & 0.64 \\
\hline
\end{tabular}


1

Fig. 1. Workflow of PHYTOGEN database process

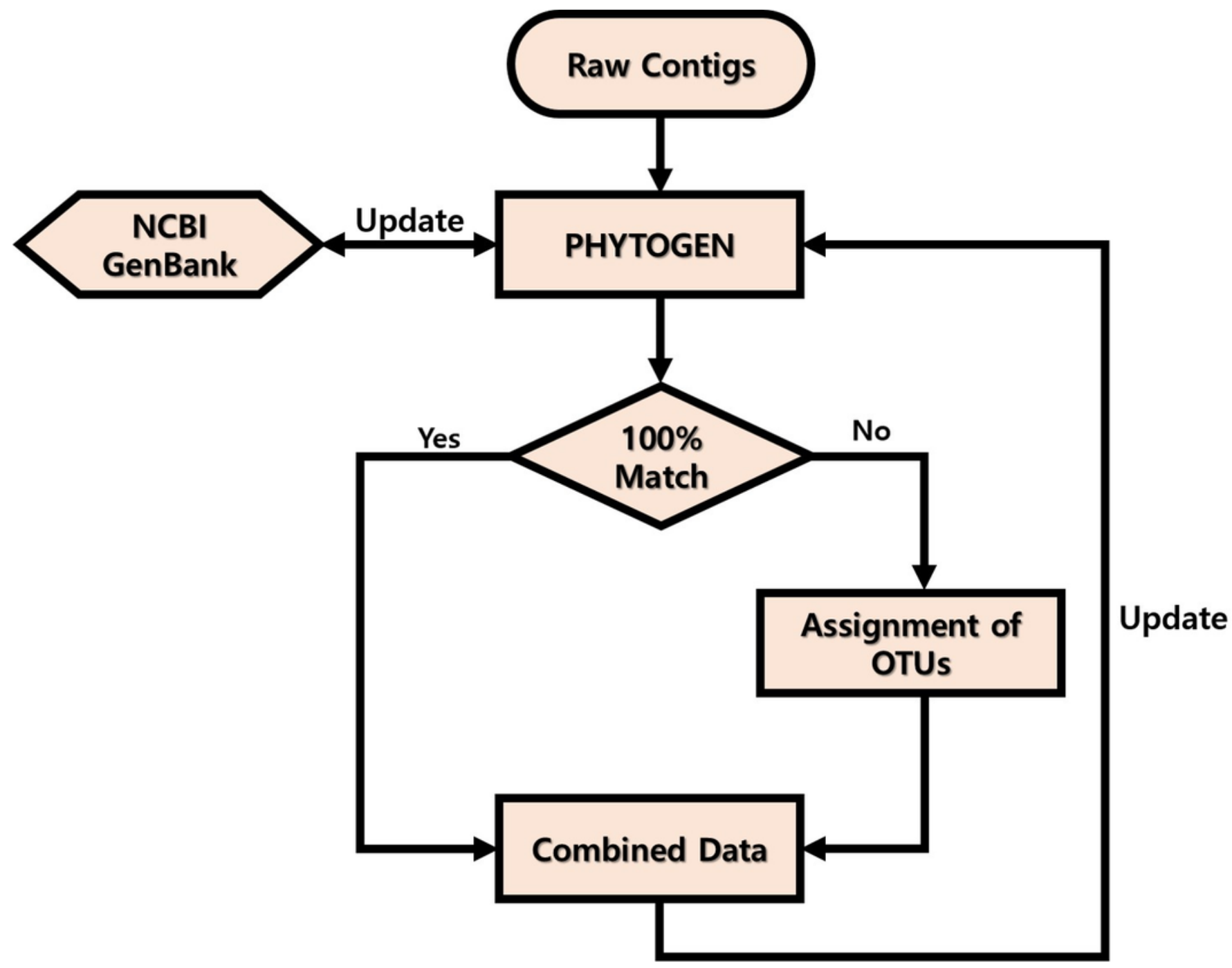


2

Fig. 2. Composition of phytoplankton by the different cut-off similarity of OTUs

(A) Each bar shows the ratio of phytoplankton phyla according to cut-off similarity from $90 \%$ to $99 \%$. (B) Each bar shows the ratio of phytoplankton classes according to cut-off similarity from $90 \%$ to $99 \%$. 
(A)

$99 \%$

$97 \%$

$95 \%$

$90 \%$

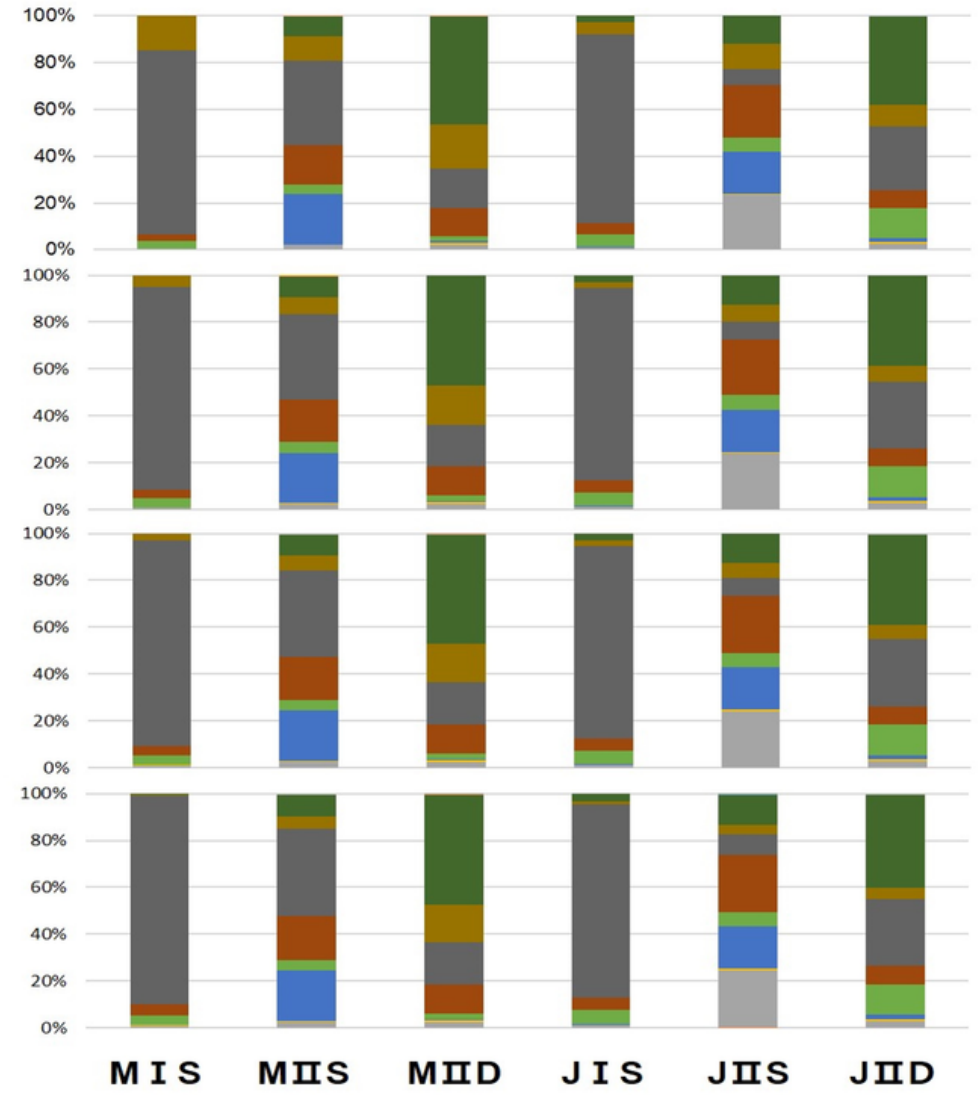

\begin{tabular}{|c|c|c|c|}
\hline Bacteria & Cercozoa & Chlorophyta & Cryptophyta \\
\hline Cyanobacteria & E Dinophyta & E Firmicutes & Eaptophyta \\
\hline E Ochrophyta & E Unclassified & Dlanctomycetes & Eroteobacteria \\
\hline Ehodophyta & Streptophyta & = Verrucomicrobia & Plant \\
\hline
\end{tabular}

(B)

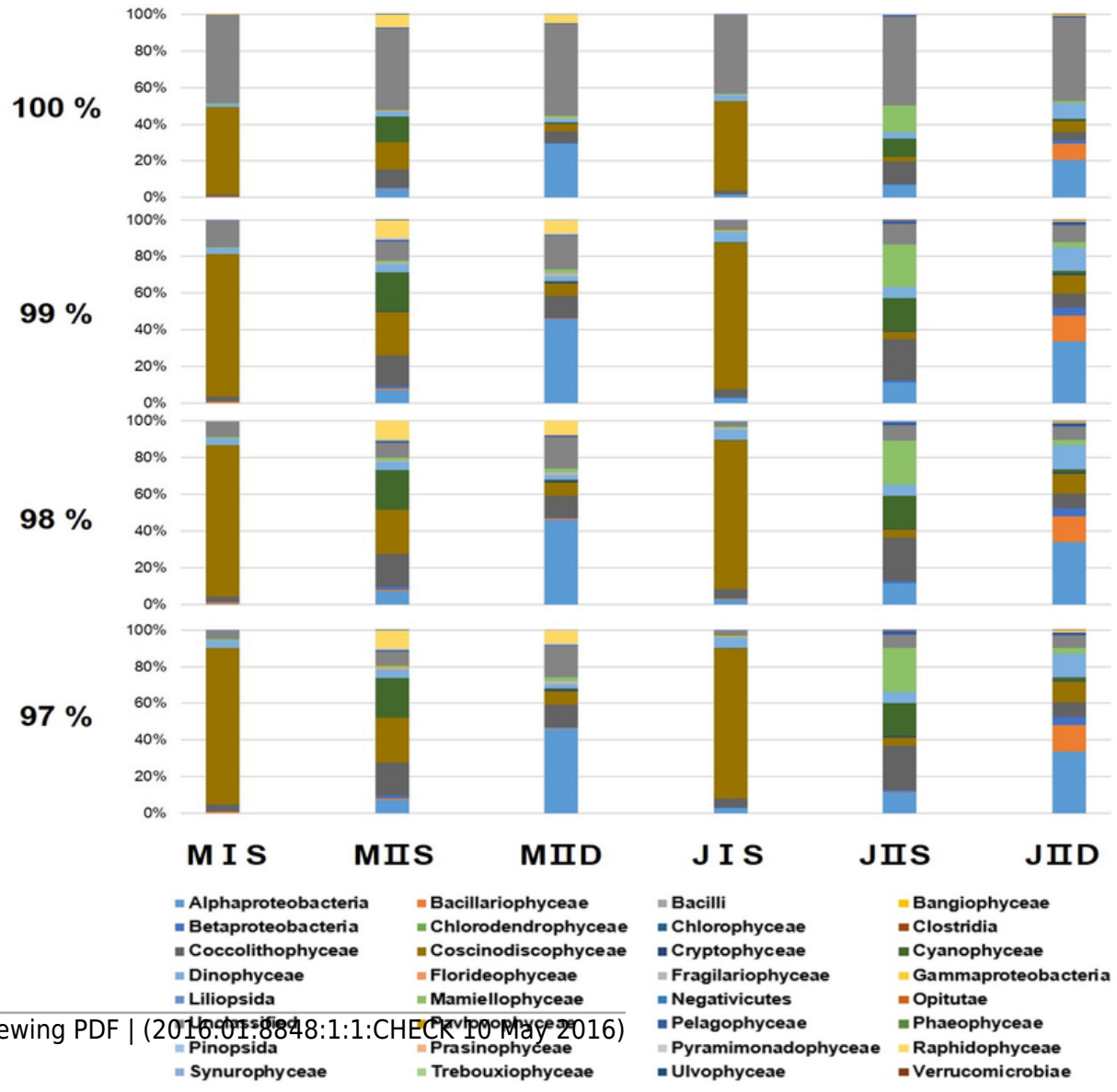


3

Fig. 3. Coverage of obtained OTUs in algal phyla

Phylogenetic tree was constructed by the Neigbhor-joining (NJ) algorithm using Molecular Evolutionary Genetics Analysis (MEGA ver 6.0). The evolutionary distances were computed using the Kimura 2-parameter method.

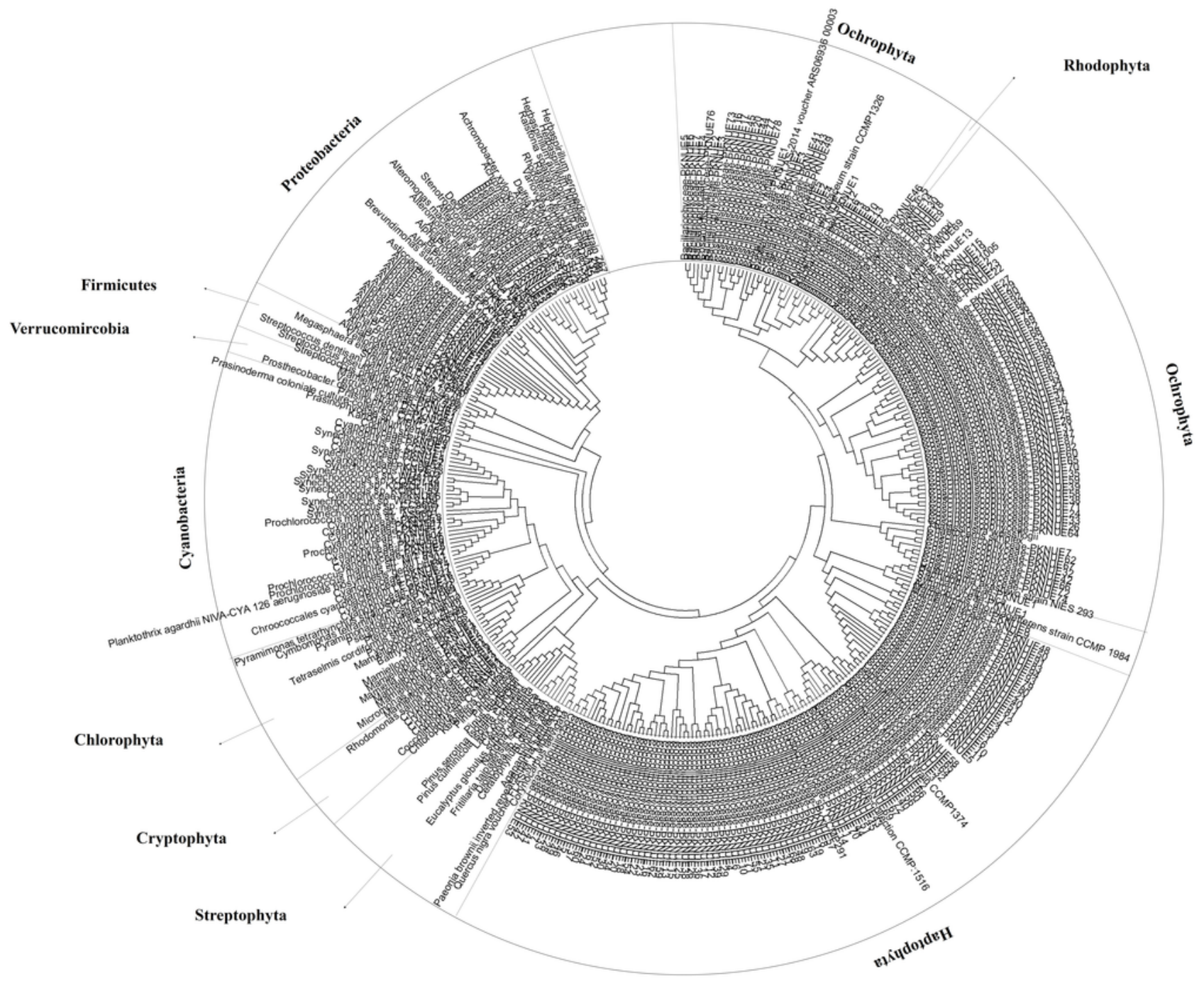


4

Fig. 4.

(A) Ratio of algal phyla in each sample with different locations, depths, and seasons Each bar shows the ratio of phytoplankton phyla according to $97 \%$ cut-off similarity (B)Venn diagram of OTUs for the four surface samples in East/Japan Sea The venn diagram was generated with Draw Venn Diagram (http://bioinformatics.psb. ugent.be/webtools/Venn/) 
(A)

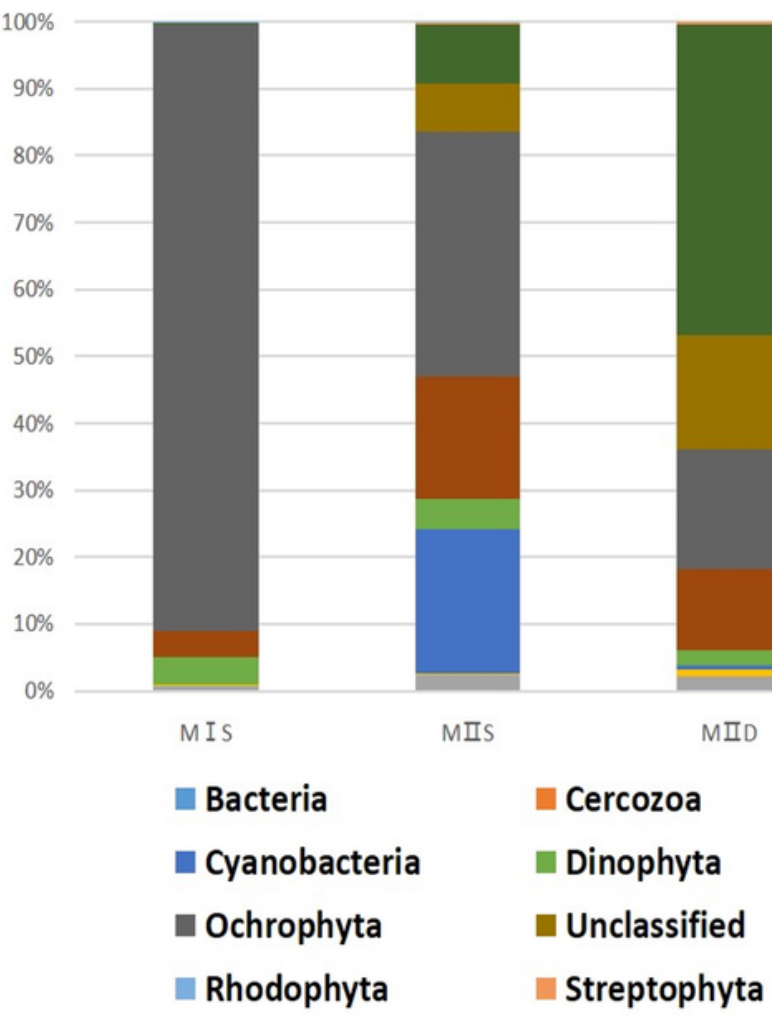

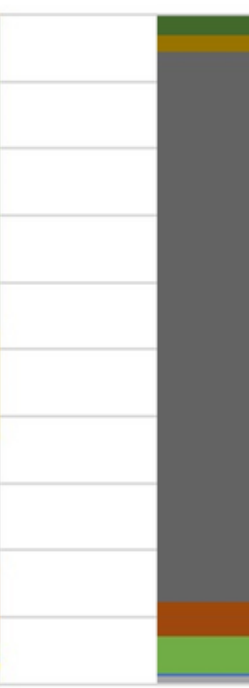

J IS

Chlorophyta

- Firmicutes

- Planctomycetes

Verrucomicrobia

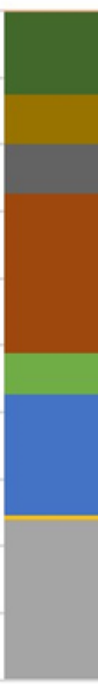

JIIS

Cryptophyta

- Haptophyta

- Proteobacteria

Plant

(B)

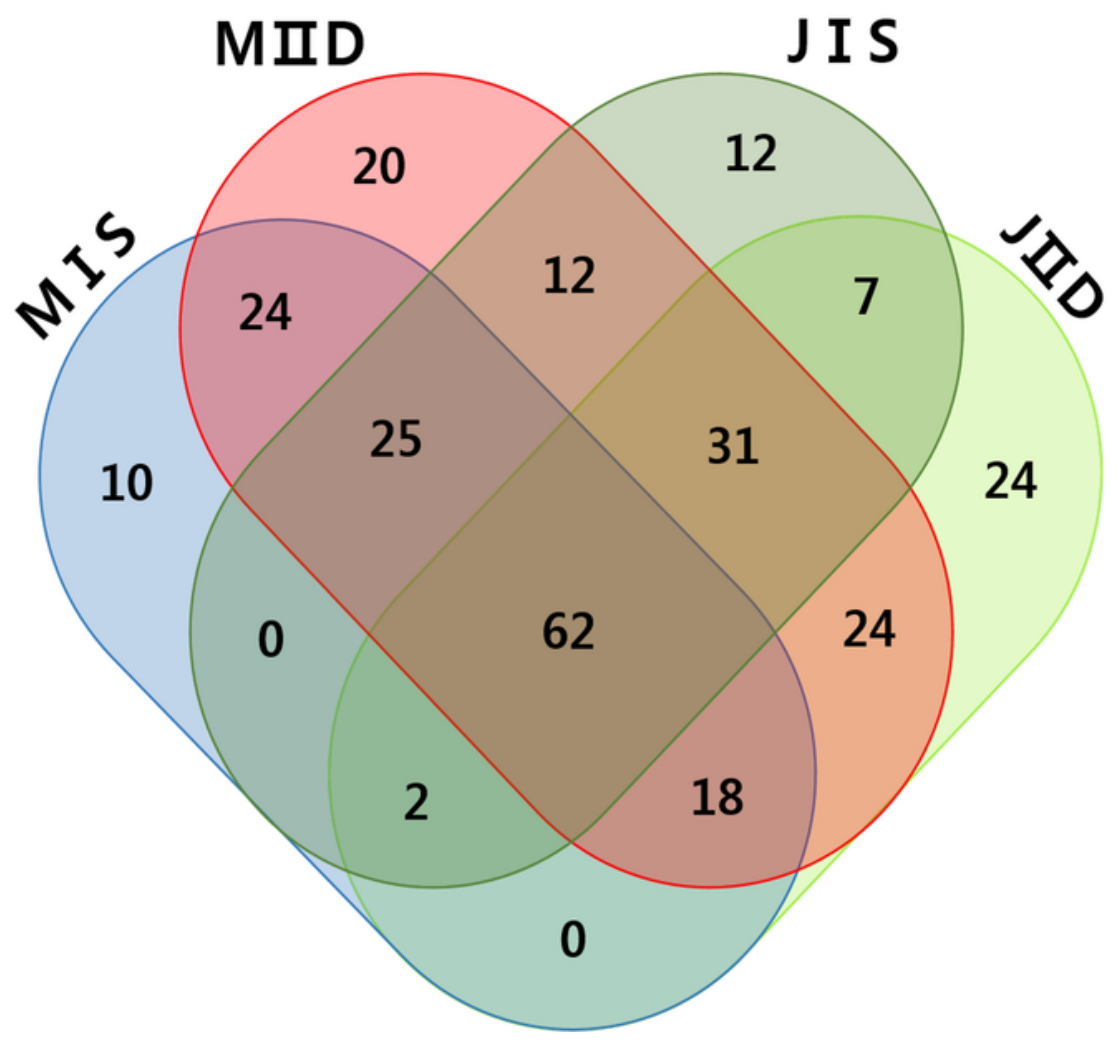




\section{Table $\mathbf{1}$ (on next page)}

Table 1. Consensus Nucleotide sequences of forward primer targeting 23S rDNA region 
1 Table 1. Consensus Nucleotide sequences of forward primer targeting 23S rDNA region

\begin{tabular}{|c|c|c|c|c|c|c|c|c|c|c|c|c|c|c|c|c|c|c|c|c|c|c|c|c|c|}
\hline A23SrVF1 & & $5^{\prime}$ & G & G & A & c & A & $R$ & A & A & A & G & A & c & c & c & $\mathrm{T}$ & A & $\mathrm{T}$ & G & & & & & \\
\hline A23SrVR1 & & & & & & c & A & $R$ & A & A & A & G & A & $\mathrm{c}$ & c & c & $T$ & A & $T$ & G & M & A & G & c & $T$ \\
\hline $\begin{array}{l}\text { P23Rv_1 } \\
\text { (Sherwood and Presting, 2007) }\end{array}$ & & $5^{\prime}$ & G & G & A & c & A & G & A & A & A & G & A & c & c & c & T & A & T & G & A & A & - & - & - \\
\hline Concensus sequence(Algae) & & & G & G & A & c & A & $R$ & A & A & A & G & $R$ & c & c & $\mathrm{Y}$ & $\mathrm{Y}$ & A & $T$ & G & M & A & $\mathrm{s}$ & c & $T$ \\
\hline Phylum & Total & & & & & & & & & & & & & & & & & & & & & & & & \\
\hline \multirow{2}{*}{ Chlorophyta } & \multirow{2}{*}{113} & & G & G & A & c & A & A & A & A & A & G & A & c & c & c & $T$ & A & $T$ & G & A & A & G & c & $\mathrm{T}$ \\
\hline & & & 113 & 113 & 113 & 113 & 113 & 113 & 113 & 113 & 113 & 113 & 113 & 113 & 113 & 113 & 113 & 113 & 113 & 113 & 113 & 113 & 113 & 113 & 113 \\
\hline \multirow{2}{*}{ Cryptophyta } & \multirow{2}{*}{\multicolumn{2}{|c|}{54}} & G & G & A & c & A & $G$ & A & A & A & G & A & c & c & c & $T$ & A & $T$ & G & A & A & G & c & $T$ \\
\hline & & & 54 & 54 & 54 & 54 & 54 & 54 & 54 & 54 & 54 & 54 & 54 & 54 & 54 & 54 & 54 & 54 & 54 & 54 & 54 & 54 & 54 & 54 & 54 \\
\hline \multirow{2}{*}{ Euglenophyta } & \multirow{2}{*}{\multicolumn{2}{|c|}{141}} & G & $G$ & A & c & A & $G$ & A & A & A & G & A & c & c & $Y(C / T)$ & $T$ & A & T & G & $M(A / C)$ & A & G & c & $T$ \\
\hline & & & 141 & 141 & 141 & 141 & 141 & 141 & 141 & 141 & 141 & 141 & 141 & 141 & 141 & $140 / 1$ & 141 & 141 & 141 & 141 & $137 / 4$ & 141 & 141 & 141 & 141 \\
\hline \multirow{2}{*}{ Ochrophyta } & \multirow{2}{*}{\multicolumn{2}{|c|}{26}} & G & G & A & c & A & G & A & A & A & G & A & c & c & c & $T$ & A & $T$ & G & A & A & G & c & $T$ \\
\hline & & & 26 & 26 & 26 & 26 & 26 & 26 & 26 & 26 & 26 & 26 & 26 & 26 & 26 & 26 & 26 & 26 & 26 & 26 & 26 & 26 & 26 & 26 & 26 \\
\hline \multirow{2}{*}{ Streptophyta } & \multirow{2}{*}{\multicolumn{2}{|c|}{198}} & G & G & A & c & A & $G$ & A & A & A & G & A & $c$ & c & $c$ & $T$ & A & $T$ & G & A & A & G & $c$ & $T$ \\
\hline & & & 198 & 198 & 198 & 198 & 198 & 198 & 198 & 198 & 198 & 198 & 198 & 198 & 198 & 198 & 198 & 198 & 198 & 198 & 198 & 198 & 198 & 198 & 198 \\
\hline \multirow{2}{*}{ Dinophyta } & \multirow{2}{*}{\multicolumn{2}{|c|}{13}} & G & G & A & c & A & $R(A / G)$ & A & A & A & G & $R(A / G)$ & c & c & c & $T$ & A & $T$ & G & A & A & $s$ & c & $T$ \\
\hline & & & 13 & 13 & 13 & 13 & 13 & $1 / 12$ & 13 & 13 & 13 & 13 & $12 / 1$ & 13 & 13 & 13 & 13 & 13 & 13 & 13 & 13 & 13 & $12 / 1$ & 13 & 13 \\
\hline \multirow[b]{2}{*}{ Cyanobacteria } & \multirow{2}{*}{\multicolumn{2}{|c|}{195}} & G & $G$ & A & c & A & G & A & A & A & G & A & c & $c$ & c & $Y(C / T)$ & A & $T$ & G & A & A & G & c & $T$ \\
\hline & & & 195 & 195 & 195 & 195 & 195 & 195 & 195 & 195 & 195 & 195 & 195 & 195 & 195 & 195 & $194 / 1$ & 195 & 195 & 195 & 195 & 195 & 195 & 195 & 195 \\
\hline \multirow{2}{*}{ Proteobacteria- $\alpha$} & \multirow{2}{*}{\multicolumn{2}{|c|}{198}} & * & G & A & c & $R(A / G)$ & G & A & A & A & $R(A / G)$ & A & c & c & c & $Y(C / T)$ & $\mathrm{R}$ & $T$ & G & $M(A / C)$ & A & c & c & $T$ \\
\hline & & & * & 198 & 198 & 198 & $1 / 197$ & 198 & 198 & 198 & 198 & 2/196 & 198 & 198 & 198 & 198 & 96/102 & 109/89 & 198 & 198 & 98/100 & 198 & 198 & 198 & 198 \\
\hline \multirow[b]{2}{*}{ Proteobacteria- $\beta$} & \multirow{2}{*}{\multicolumn{2}{|c|}{198}} & $\star$ & G & A & $c$ & G & G & A & A & A & G & A & c & c & c & $T$ & A & $T$ & G & A & A & c & c & $T$ \\
\hline & & & * & 198 & 198 & 198 & 198 & 198 & 198 & 198 & 198 & 198 & 198 & 198 & 198 & 198 & 198 & 198 & 198 & 198 & 198 & 198 & 198 & 198 & 198 \\
\hline \multirow{2}{*}{ Proteobacteria- $\gamma$} & \multirow{2}{*}{\multicolumn{2}{|c|}{198}} & * & $G$ & A & c & G & G & A & A & A & G & A & c & c & c & $T$ & $\mathrm{R}$ & $T$ & G & $M(A / C)$ & A & c & c & $T$ \\
\hline & & & * & 198 & 198 & 198 & 198 & 198 & 198 & 198 & 198 & 198 & 198 & 198 & 198 & 198 & 198 & $1 / 197$ & 198 & 198 & $181 / 17$ & 198 & 198 & 198 & 198 \\
\hline \multirow[t]{2}{*}{ Proteobacteria-8 } & \multirow{2}{*}{\multicolumn{2}{|c|}{151}} & * & G & $R(A / G)$ & $M(C / A)$ & $R(A / G)$ & G & $R(A / G)$ & A & A & $R(A / G)$ & R & $M(A / C)$ & c & c & $Y(C / T)$ & $\underset{(A / G / C / T)}{N}$ & $\mathrm{~K}(\mathrm{G} / \mathrm{T})$ & $\begin{array}{c}D \\
(A / G / T)\end{array}$ & $\begin{array}{c}V \\
(A / G / C)\end{array}$ & $W(A / T)$ & $M(A / C)$ & $Y(C / T)$ & $Y(C / T)$ \\
\hline & & & & 151 & $147 / 4$ & $147 / 4$ & $21 / 126$ & 151 & $147 / 4$ & 151 & 151 & $4 / 147$ & $147 / 4$ & $4 / 147$ & 151 & 151 & $32 / 119$ & $15 / 132 / 2 / 2$ & $9 / 142$ & $5 / 142 / 4$ & $90 / 7 / 54$ & $143 / 8$ & $4 / 147$ & $146 / 5$ & 4/147 \\
\hline & 198 & & * & G & A & G & G & $G$ & $R(A / G)$ & $R(A / G)$ & A & G & A & c & c & c & T & G & $T$ & G & $s(C / G)$ & A & c & c & $\mathrm{T}$ \\
\hline Proteobacteria- $\varepsilon$ & 198 & & * & 198 & 198 & 198 & 198 & 198 & $196 / 2$ & $190 / 8$ & 198 & 198 & 198 & 198 & 198 & 198 & 198 & 198 & 198 & 198 & $150 / 48$ & 198 & 198 & 198 & 198 \\
\hline
\end{tabular}




\section{Table 2 (on next page)}

Table 2. Comparison of modified primer and original primer experiment 
1 Table 2. Comparison of modified primer and original primer experiment

\begin{tabular}{|c|c|c|c|c|c|c|}
\hline \multirow{2}{*}{ Phylum } & \multicolumn{3}{|c|}{ Modified Primers } & \multicolumn{3}{|c|}{ Original Primers } \\
\hline & Amplicons & $\%$ & OTUs & Amplicons & $\%$ & OTUs \\
\hline Ochrophyta & 398,588 & 63.68 & 76 & 24,006 & 12.92 & 31 \\
\hline Unclassified & 122,178 & 19.52 & - & 81,562 & 43.90 & - \\
\hline Haptophyta & 47,816 & 7.64 & 22 & 2,198 & 1.18 & 6 \\
\hline Proteobacteria & 28,499 & 4.55 & 2 & 76,271 & 41.06 & 22 \\
\hline Dinophyta & 28,008 & 4.47 & 5 & 1,510 & 0.81 & 3 \\
\hline Chlorophyta & 828 & 0.13 & 1 & 226 & 0.12 & 1 \\
\hline Total & 625,917 & 100 & 106 & 185,773 & 100 & 63 \\
\hline
\end{tabular}

3

4 


\section{Table 3(on next page)}

Table 3. Summary of end-paired phytoplanktonic contigs and processed OTUs in samples of East/Japan Sea 
1 Table 3. Summary of end-paired phytoplanktonic contigs and processed OTUs in samples of East/Japan Sea

\begin{tabular}{|c|c|c|c|c|c|c|c|c|c|c|c|c|c|c|c|c|c|c|}
\hline \multirow{2}{*}{ Phylum } & \multicolumn{3}{|c|}{ M I S } & \multicolumn{3}{|c|}{ MIIS } & \multicolumn{3}{|c|}{ MID } & \multicolumn{3}{|c|}{ J I S } & \multicolumn{3}{|c|}{ JIIS } & \multicolumn{3}{|c|}{ JIID } \\
\hline & Contigs & $\begin{array}{c}\text { Ratio } \\
((\%)\end{array}$ & OTUs & Contigs & $\begin{array}{c}\text { Ratio } \\
(\%)\end{array}$ & OTUs & Contigs & $\begin{array}{c}\text { Ratio } \\
(\%)\end{array}$ & OTUs & Contigs & $\begin{array}{c}\text { Ratio } \\
(\%)\end{array}$ & OTUs & Contigs & $\begin{array}{c}\text { Ratio } \\
(\%)\end{array}$ & OTUs & Contigs & $\begin{array}{c}\text { Ratio } \\
(\%)\end{array}$ & OTUs \\
\hline Chlorophyta & 4,815 & 0.75 & 21 & 7,261 & 2.30 & 22 & 8,656 & 2.26 & 15 & 7,036 & 1.13 & 13 & 98,420 & 23.91 & 19 & 8,279 & 2.85 & 14 \\
\hline Cryptophyta & 1,063 & 0.17 & 6 & 1,151 & 0.36 & 5 & 3,684 & 0.96 & 4 & 24 & 0.00 & 4 & 2,839 & 0.69 & 4 & 1,878 & 0.65 & 5 \\
\hline Cyanobacteria & 209 & 0.03 & 17 & 67,933 & 21.54 & 30 & 2,029 & 0.53 & 19 & 2,561 & 0.41 & 15 & 74,420 & 18.08 & 31 & 5,264 & 1.81 & 26 \\
\hline Dinophyta & 24,441 & 3.82 & 19 & 14,173 & 4.49 & 16 & 8,919 & 2.33 & 15 & 35,103 & 5.65 & 14 & 24,917 & 6.05 & 16 & 37,744 & 13.01 & 17 \\
\hline Firmicutes & - & 0.00 & 0 & 2 & 0.00 & 2 & - & 0.00 & 0 & - & 0.00 & 0 & 38 & 0.01 & 2 & 123 & 0.04 & 2 \\
\hline Haptophyta & 24,094 & 3.77 & 74 & 57,385 & 18.20 & 75 & 46,724 & 12.22 & 73 & 31,230 & 5.03 & 70 & 98,841 & 24.01 & 76 & 22,534 & 7.77 & 75 \\
\hline Ochrophyta & 552,850 & 86.40 & 118 & 115,611 & 36.66 & 119 & 67,856 & 17.74 & 113 & 510,993 & 82.31 & 109 & 30,117 & 7.32 & 118 & 82,463 & 28.43 & 123 \\
\hline Unclassified & 30,246 & 4.73 & $\mathrm{~N} / \mathrm{A}$ & 22,399 & 7.10 & N/A & 65,121 & 17.03 & N/A & 14,556 & 2.34 & N/A & 30,478 & 7.40 & $\mathrm{~N} / \mathrm{A}$ & 19,497 & 6.72 & N/A \\
\hline Proteobacteria & 1,177 & 0.18 & 19 & 27,885 & 8.84 & 46 & 178,140 & 46.57 & 41 & 17,715 & 2.85 & 32 & 50,790 & 12.34 & 46 & 111,346 & 38.38 & 54 \\
\hline Rhodophyta & 772 & 0.12 & 1 & 315 & 0.10 & 1 & 16 & 0.00 & 1 & 2 & 0.00 & 1 & 22 & 0.01 & 1 & 28 & 0.01 & 1 \\
\hline Streptophyta & 52 & 0.01 & 4 & 424 & 0.13 & 4 & 1,103 & 0.29 & 8 & 422 & 0.07 & 6 & 404 & 0.10 & 9 & 7 & 0.00 & 3 \\
\hline Verrucomicrobia & 117 & 0.02 & 2 & 807 & 0.26 & 2 & 253 & 0.07 & 2 & 1,200 & 0.19 & 2 & 312 & 0.08 & 3 & 923 & 0.32 & 4 \\
\hline Plant & - & 0.00 & 0 & 1 & 0.00 & 1 & - & 0.00 & 0 & - & 0.00 & 0 & - & 0.00 & 0 & - & 0.00 & 0 \\
\hline Total & 639,836 & 100 & 281 & 315,347 & 100 & 323 & 382,501 & 100 & 291 & 620,842 & 100 & 266 & 411,598 & 100 & 325 & 290,086 & 100 & 324 \\
\hline
\end{tabular}




\section{Table 4(on next page)}

Table 4. Top 20 phytoplanktonic OTUs in each sample of East/Japan Sea 
1 Table 4. Top 20 phytoplanktonic OTUs in each sample of East/Japan Sea

\begin{tabular}{|c|c|c|c|c|c|c|c|c|c|c|c|}
\hline M IS & & MIIS & & $\mathrm{MID}$ & & JIS & & JIIS & & $\mathrm{JIID}$ & \\
\hline otUs & $\%$ & отUs & $\%$ & otUs & $\%$ & oTUs & $\%$ & otUs & $\%$ & otUs & $\%$ \\
\hline Coscinodiscophyceae-PKNUE1 & 58.34 & Cyanophyceae-PKNUE1 & 14.21 & Heterosigma akashiwo - EU168190 & 7.47 & Coscinodiscophyceae-PKNUE52 & 23.33 & Mamiellophyceae-PKNUE1 & 7.06 & Bacillariophyceae-PKNUE4 & 12.93 \\
\hline Coscinodiscophyceae-PKNUE2 2 & 2.63 & Coscinodiscophyceae-PKNUE1 & 11.79 & Coccolithophyceae-PKNUE71 & 3.20 & Coscinodiscophyceae-PKNUE55 & 16.61 & Synechococcus sp. - CP000110 & 6.48 & Dinophyceae-PKNUE10 & 10.89 \\
\hline Coscinodiscophyceae-PKNUE79 & 2.56 & Heterosigma akashiwo - EU168190 & 9.91 & Fragilariophycea-PKNUE1 & 1.90 & Coscinodiscophyceae-PKNUE25 & 9.04 & Dinophyceae-PKNUE10 & 3.84 & Coccolithophycea-PKNUE4 & 2.49 \\
\hline Dinophyceae-PKNUE7 & 1.63 & Synechococcus sp. - CP000097 & 5.04 & Phaeocystis antarctica - JN117275 & 1.24 & Coscinodiscophyceae-PKNUE56 & 4.67 & Coccolithophyceae-PKNUE4 & 3.59 & Mamiellophyceae-PKNUE8 & 1.58 \\
\hline Coscinodiscophyceae-PKNUE49 & 0.99 & Coccolithophyceae-PKNUE42 & 1.94 & Coscinodiscophyceae-PKNUE92 & 0.89 & Coscinodiscophyceae-PKNUE57 & 3.69 & Micromonas pusilla - FN563097 & 3.37 & Coscinodiscophyceae-PKNUE55 & 1.46 \\
\hline Coscinodiscophyceae-PKNUE41 & 0.89 & Dinophyceae-PKNUE10 & 1.93 & Coscinodiscophyceae-PKNUE1 & 0.83 & Coscinodiscophyceae-PKNUE58 & 2.18 & Prochlorococcus marinus - CP000576 & 3.34 & Coscinodiscophyceae-PKNUE74 & 1.34 \\
\hline Coscinodiscophyceae-PKNUE4 & 0.87 & Coccolithophyceae-PKNUE5 & 1.81 & Coscinodiscophyceae-PKNUE51 & 0.73 & Dinophyceae-PKNUE4 & 2.07 & Mamiellophycea-PKNUE3 & 3.04 & Coscinodiscophyceae-PKNUE90 & 1.22 \\
\hline Coscinodiscophyceae-PKNUE3 & 0.76 & Coccolithophyceae-PKNUE6 & 1.08 & Cryptophyceae-PKNUE1 & 0.69 & Coscinodiscophyceae-PKNUE59 & 1.87 & Mamiellophyceae-PKNUE6 & 3.02 & Dinophyceae-PKNUE6 & 1.21 \\
\hline Coscinodiscophyceae-PKNUE40 & 0.72 & Coscinodiscophyceae-PKNUE13 & 1.06 & Coccolithophyceae-PKNUE50 & 0.68 & Dinophyceae-PKNUE10 & 1.80 & Synechococcus sp. - CP006882 & 2.40 & Coccolithophyceae-PKNUE38 & 1.18 \\
\hline Coscinodiscophyceae-PKNUE19 & 0.65 & Dinophyceae-PKNUE7 & 1.04 & Dinophyceae-PKNUE11 & 0.64 & Coscinodiscophyceae-PKNUE60 & 1.67 & Mamiellophyceae-PKNUE2 & 2.18 & Mamiellophyceae-PKNUE1 & 1.09 \\
\hline Coscinodiscophyccae-PKNUE91 & 0.62 & Coscinodiscophyceae-PKNUE2 & 1.02 & Mamiellophyceae-PKNUE6 & 0.54 & Coscinodiscophyceae-PKNUE26 & 1.29 & Mamiellophyceac-PKNUE4 & 1.87 & Coccolithophyceae-PKNUE7 & 1.03 \\
\hline Coscinodiscophyceae-PKNUE5 & 0.58 & Coscinodiscophyceae-PKNUE90 & 0.98 & Mamiellophyceae-PKNUE8 & 0.50 & Dinophyceae-PKNUE7 & 1.05 & Mamiellophyceae-PKNUE8 & 1.78 & Coscinodiscophyceae-PKNUE18 & 1.01 \\
\hline Coscinodiscophyceae-PKNUE6 & 0.51 & Coccolithophyceae-PKNUE40 & 0.91 & Coccolithophyceae-PKNUE73 & 0.49 & Coscinodiscophyceae-PKNUE27 & 0.98 & Coccolithophyceae-PKNUE50 & 1.69 & Coscinodiscophyceae-PKNUE52 & 0.74 \\
\hline Coscinodiscophyceae-PKNUE80 & 0.50 & Coscinodiscophyceae-PKNUE51 & 0.84 & Coccolithophyceae-PKNUE47 & 0.49 & Coscinodiscophyceae-PKNUE 82 & 0.93 & Dinophyceae-PKNUE12 & 1.62 & Coscinodiscophyceae-PKNUE76 & 0.73 \\
\hline Dinophyceae-PKNUE8 & 0.45 & Coccolithophyceae-PKNUE43 & 0.82 & Coccolithophycea-PKNUE4 & 0.47 & Coscinodiscophyceae-PKNUE28 & 0.83 & Synechococcus sp. - CP000097 & 1.45 & Coscinodiscophyceae-PKNUE89 & 0.71 \\
\hline Coscinodiscophyceae-PKNUE8 & 0.43 & Mamiellophyceae-PKNUE8 & 0.77 & Coscinodiscophyceae-PKNUE24 & 0.47 & Coscinodiscophyceae-PKNUE1 & 0.67 & Coccolithophyceae-PKNUE21 & 1.25 & Coscinodiscophyceae-PKNUE75 & 0.69 \\
\hline Bacillariophyceae-PKNUE2 & 0.42 & Coccolithophyceae-PKNUE1 & 0.76 & Mamiellophyceae-PKNUE1 & 0.46 & Coscinodiscophyceae-PKNUE45 & 0.66 & Coscinodiscophyceae-PKNUE94 & 1.23 & Cyanophyceae-PKNUE12 & 0.68 \\
\hline Coscinodiscophyceae-PKNUE21 & 0.40 & Cocolithophyceae-PKNUE44 & 0.72 & Coscinodiscophyceae-PKNUE52 & 0.46 & Coscinodiscophyceae-PKNUE61 & 0.59 & Mamiellophyceae-PKNUE5 & 1.09 & Coccolithophyceae-PKNUE64 & 0.66 \\
\hline Coscinodiscophyceae-PKNUE48 & 0.40 & Fragilariophyceac-PKNUE1 & 0.68 & Coscinodiscophyceae-PKNUE20 & 0.43 & Coscinodiscophyceae-PKNUE62 & 0.55 & Coccolithophyceae-PKNUE54 & 0.84 & Coccolithophyceae-PKNUE46 & 0.65 \\
\hline Dinophyceae-PKNUE1 & 0.40 & Coscinodiscophyceae-PKNUE92 & 0.68 & Coccolithophyceae-PKNUE15 & 0.42 & Coscinodiscophyceae-PKNUE63 & 0.54 & Cyanophyceae-PKNUE5 & 0.69 & Bacillariophyceae-PKNUE5 & 0.64 \\
\hline
\end{tabular}

